\title{
Sobre existência de estados de equilíbrio e limite em temperatura zero para shifts de Markov topologicamente mixing
}

\author{
Victor Andres Vargas Cubides
}

TESE APRESENTADA

$\mathrm{AO}$

Instituto De Matemática e Estatística

DA

Universidade DE SÃo PAUlo

PARA

OBTENÇÃO DO TÍTULO

$\mathrm{DE}$

DOUTOR EM CIÊNCIAS

Programa: Matemática

Orientador: Prof. Dr. Ricardo dos Santos Freire Jr.

Durante o desenvolvimento deste trabalho o autor recebeu auxílio financeiro da CAPES

São Paulo, Outubro 2015 


\section{Sobre existência de estados de equilíbrio e limite em temperatura zero para shifts de Markov topologicamente mixing}

Esta versão da tese contém as correções e alterações sugeridas pela comissão julgadora durante a defesa da versão original do trabalho realizada em 16/10/2015. Uma copia da versão original está disponível no Instituto de Matemática e Estatística da Universidade de São Paulo.

Comissão Julgadora:

- Prof. Dr. Ricardo dos Santos Freire Jr. - USP

- Prof. Dr. Rodrigo Bissacot Proença - USP

- Prof. Dr. Eduardo Garibaldi - UNICAMP

- Prof. Dr. Renaud Leplaideur - Université de Brest

- Prof. Dr. Artur Oscar Lopes - UFRGS 


\section{Agradecimentos}

Gostaria em primeiro lugar de agradecer a meus pais Raul y Gloria, sem seu apoio incondicional não teria sido possível chegar até aqui, também quero agradecer a minha irmã Jeimy, ela é um exemplo de dedicação e bom comportamento que sempre me inspira a melhorar, não posso esquecer agradecer a meus colegas e mais que isso amigos César e Elkin pelo seu apoio no momento certo. Também queria agradecer a meu orientador Ricardo por seu acompanhamento ao longo deste processo, seus aportes foram fundamentais para a culminação deste trabalho, ao professor Rodrigo por suas contribuições que ajudaram guiar o trabalho nesta direção, a todos esses professores que de algum modo ajudaram na minha formação durante meu doutorado, à Universidade de São Paulo pelo seu acolhimento, à CAPES pelo seu apoio financeiro o qual foi de grande ajuda durante a minha permanência no Brasil e além disso tem ajudado à divulgação deste trabalho fora da universidade, e por último a todas essas pessoas que fizeram um esforço por me tolerar nesses momentos de altos níveis de estresse. 


\section{Resumo}

VARGAS, V. A. Sobre existência de estados de equilíbrio e limite em temperatura zero para shifts de Markov topologicamente mixing. 2015. 59 f. Tese Doutorado - Instituto de Matemática e Estatística, Universidade de São Paulo, São Paulo, 2015

O objetivo desta tese é demonstrar que para um subshift de Markov topologicamente transitivo com alfabeto enumerável e um potencial $f$ com pressão de Gurevic finita e $\bar{V}(f)<\infty$, existe um único estado de equilíbrio $\mu_{t f}$ para cada $t>1$, e a família $\left(\mu_{t f}\right)_{t>1}$ tem um ponto de acumulação quando $t \rightarrow \infty$. Além disso se também supomos que o $f$ é um potencial de Markov, demonstramos que a família de estados de equilíbrio $\left(\mu_{t f}\right)_{t>1}$ converge quando $t \rightarrow \infty$. Finalmente demonstramos a continuidade em $\infty$ da entropia com respeito ao parâmetro $t$. Estos resultados não dependem da hipótese de existência de medidas de Gibbs.

Palavras-chave: estados de equilíbrio, estados de Gibbs, potenciais somáveis, potenciais de Markov, subshifts de Markov, limite em temperatura zero, medidas maximizantes. 


\section{Abstract}

VARGAS, V. A. On equilibrium states existence and zero temperature limit for topologically mixing Markov shifts. 2015. 59 p. PhD Thesis - Mathematics and Statistics Institute, Sao Paulo University, Sao Paulo, 2015.

The aim of this thesis is to prove that for a topologically transitive Markov subshift with countable alphabet and a summable potential $f$ with finite Gurevic pressure and $\bar{V}(f)<\infty$, there exists an equilibrium state $\mu_{t f}$ for each $t>1$, and the family $\left(\mu_{t f}\right)_{t>1}$ has an accumulation point as $t \rightarrow \infty$. Moreover if we also assume that $f$ is a Markov potential we prove that the equilibrium states family $\left(\mu_{t f}\right)_{t>1}$ converges as $t \rightarrow \infty$. Finally we prove the continuity at $\infty$ of the entropy with respect to the parameter $t$. These results do not depend on assuming the existence of Gibbs measures.

Keywords: equilibrium states, Gibbs states, summable potentials, Markov potentials, Markov subshifts, zero temperature limit, maximizing measures. 


\section{Sumário}

$\begin{array}{ll}\text { Lista de Símbolos } & \text { ix }\end{array}$

1 Introdução $\quad 1$

$\begin{array}{lll}2 & \text { Preliminares } & 7\end{array}$

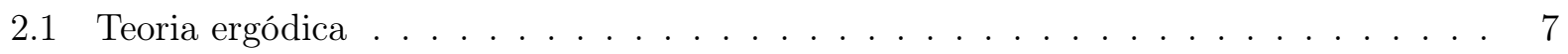

2.2 Formalismo termodinâmico f . . . . . . . . . . . . . . . . . . 12

3 Operador de Ruelle e estados de equilíbrio $\quad 17$

3.1 Operador de Ruelle . . . . . . . . . . . . . . . . . . . . . . . 17

3.2 Unicidade dos estados de equilíbrio . . . . . . . . . . . . . . 26

4 Limite em temperatura zero sobre compactos e otimização ergódica 33

4.1 Otimização ergódica . . . . . . . . . . . . . . . . . 33

4.2 Limite em temperatura Zero para subshifts compactos . . . . . . . . . . . . 37

5 Limite em temperatura Zero para subshifts não compactos 43

5.1 Aproximação por subshifts compactos . . . . . . . . . . . . . . . . 44

5.2 Demonstração do teorema A . . . . . . . . . . . . . . . . 47

5.3 Demonstração dos teoremas B e C . . . . . . . . . . . . . . . . . . 53

6 Conclusões $\quad 59$

$\begin{array}{ll}\text { Referências Bibliográficas } & 61\end{array}$

$\begin{array}{ll}\text { Índice Remissivo } & 63\end{array}$ 


\title{
Lista de Símbolos
}

\author{
$\mathcal{M}(X) \quad$ Conjunto das medidas de probabilidade sobre $X$ \\ $\mathcal{M}_{T}(X) \quad$ Conjunto das medidas de probabilidade $T$-invariantes sobre $X$ \\ $\mathcal{M}_{\text {erg }}(X)$ Conjunto das medidas de probabilidade ergódicas sobre $X$ \\ $\mathcal{M}_{\text {per }}(X)$ Conjunto das medidas de probabilidade periódicas sobre $X$ \\ $\mathcal{M}_{\text {max }}(f)$ Conjunto das medidas de probabilidade $f$-maximizantes \\ $h(\mu) \quad$ Entropia da medida $\mu$ \\ $P_{\mu}(f) \quad$ Pressão métrica do potencial $f$ com respeito à $\mu$ \\ $P_{\text {top }}(f) \quad$ Pressão topológica do potencial $f$ \\ $P_{G}(f) \quad$ Pressão de Gurevic do potencial $f$ \\ $\mu_{f} \quad$ Estado de equilíbrio associado ao potencial $f$
}




\section{Capítulo 1}

\section{Introdução}

Seja $X$ um espaço Hausdorff e $T: X \rightarrow X$ uma transformação sobrejetiva, então as compostas da transformação $T$ definem uma $\mathbb{N}$-ação sobre o conjunto $X$ a qual é um sistema dinâmico discreto que usualmente é denotado pela dupla $(T, X)$. A teoria ergódica pretende estudar o comportamento das medidas que satisfazem a relação $\mu_{*} T=\mu$, onde $\mu_{*} T(E)=\mu\left(T^{-1}(E)\right)$ para cada conjunto mensurável $E \subset X$, além disso busca fornecer informação quantitativa das orbitas geradas pela dinâmica $(T, X)$ para quase todo ponto do espaço $X$.

A área começo a ser estudada no século XIX e tem seus origens na mecânica estatística, nessa época os físicos L. Boltzmann e H. Poincaré estavam interessados no estudo da cinética de gases modelados por fluxos Hamiltonianos, porém encontrar soluções às equações diferenciáveis relacionadas com esse tipo de fluxos ficou trabalhoso de mais, nesse momento surgiu uma ideia do H. Poincaré a qual consistiu em discretizar o problema e estudar o comportamento do conjunto errante, ele concluiu que tinha volume zero, esse é um dos teoremas fundamentais da área e poderia ser considerado o origem da teoria ergódica, no entanto quando eles começaram a estudar esse problema acreditaram que as medias temporais ao longo das orbitas coincidiam com as medias espaciais, mas depois deram conta que essa propriedade não é necessariamente certa no caso geral, porém depois de dar conta desse erro chamaram os sistemas nos quais essa condição é satisfeita de sistemas ergódicos.

A maior parte do desenvolvimento da teoria ergódica foi no século $X X$, alguns dos matemáticos que fizeram as maiores contribuições no desenvolvimento inicial desta área foram G. D. Birkhoff, A. Kolmogorov, J. Von Neumann e Y. Sinai. Já na década dos 70's o físico D. Ruelle e o matemático R. Bowen começaram estudar um ramo da teoria ergódica conhecido como formalismo termodinâmico, eles queriam estudar as propriedades de medidas invariantes que maximizam a pressão $P_{\mu}(f)=h(\mu)+\int f d \mu$, onde $f$ é uma função contínua chamada de potencial e $h(\mu)$ é a entropia de 
Kolmogorov-Sinai, essas medidas foram chamadas de estados de equilíbrio associados ao potencial $f$. Já nos finais da década dos 90's e começos do século XXI os matemáticos O. Jenkinson R. Mauldin, O. Sarig e M. Urbański entre outros, começaram a estudar formalismo termodinâmico no contexto de subshifts de Markov com alfabeto enumerável e tiveram alguns dos mais importantes avanços nessa linha.

No caso que para cada $t>1$ existe um único estado de equilíbrio $\mu_{t f}$ associado ao potencial $t f$, um problema interessante é estudar existência de pontos de acumulação da família $\left(\mu_{t f}\right)_{t>1}$ quando $t \rightarrow \infty$, isso porque esses pontos de acumulação são medidas maximizantes para o potencial $f$, além disso porque desde o ponto de vista da mecânica estatística, num sistema dinâmico $(T, X)$ com iteração entre partículas dada pelo potencial $f$, a temperatura é representada pelo parâmetro $\frac{1}{t}$, portanto existência de pontos de acumulação dessa família de estados de equilíbrio é associada à não existência de transições de fase do sistema quando se tem congelamento, na física é possível interpretar isso como não ter nenhuma perda das propriedades dos materiais em baixas temperaturas. No caso de existir o limite da família $\left(\mu_{t f}\right)_{t>1}$ quando $t \rightarrow \infty$ é chamado de limite em temperatura zero. Em geral o estudo do comportamento desses pontos de acumulação é conhecido como problemas de limites em temperatura zero e a sua importância é baseada na ligação que faz entre o formalismo termodinâmico e a otimização ergódica.

O objetivo deste trabalho é mostrar existência dos estados de equilíbrio associados a cada um dos elementos de uma família de potenciais $(t f)_{t>1}$ que satisfaz algumas propriedades desejáveis, além disso demonstramos existência de pontos de acumulação da família $\left(\mu_{t f}\right)_{t>1}$ quando $t \rightarrow \infty$ e no caso que o potencial é Markov queremos demonstrar a existência do limite em temperatura zero, também estudaremos o comportamento da família das entropias desses estados de equilíbrio $\left(h\left(\mu_{t f}\right)\right)_{t>1}$ quando $t \rightarrow \infty$.

Alguns trabalhos já foram feitos nessa direção, porém neles é usada a propriedade que o subshift é finitamente primitivo, a ideia é enfraquecer um pouco essa propriedade para o caso onde a dinâmica é topologicamente transitiva.

Seja $f$ um potencial, para cada $n \geq 1$ definimos a $n$-ésima variação do $f$ como

$$
V_{n}(f):=\sup \left\{|f(x)-f(y)|: x_{0} \ldots x_{n-1}=y_{0} \ldots y_{n-1}\right\}
$$

e vamos definir $\bar{V}(f):=\sum_{n \geq 1} V_{n}(f)$.

Nosso primeiro resultado garante existência dos estados de equilíbrio para uma família de potenciais dependendo do parâmetro $t$, o teorema é o seguinte: 
Teorema A. Seja $\Sigma$ um subshift de Markov topologicamente transitivo e $f$ um potencial somável, com pressão topológica finita e $\bar{V}(f)<\infty$. Então para cada $t>1$ existe um estado de equilíbrio $\mu_{t f}$ associado ao potencial $t f$, além disso a família $\left(\mu_{t f}\right)_{t>1}$ tem um ponto de acumulação $\mu_{\infty}$ quando $t \rightarrow \infty$.

A demonstração deste resultado é similar do que um resultado que aparece demonstrado em [14] que garante usando hipóteses similares existência de uma automedida associada ao operador adjunto do operador de Ruelle, porém é conhecido que no caso não-compacto essa automedida não é necessariamente um estado de equilíbrio, portanto o ganho deste teorema é garantir a existência da família de estados de equilíbrio no caso topologicamente transitivo.

No caso que o alfabeto é finito, existência de pontos de acumulação é imediata, porém a existência do limite em temperatura zero não é trivial. O primeiro trabalho nesta direção foi apresentado por Brémont em [5], nesse trabalho ele demonstrou existência do limite em temperatura zero no caso de potenciais localmente constantes definidos sobre subshifts com alfabeto finito, depois disso Leplaideur em [13] conseguiu mostrar uma forma explícita do limite em temperatura zero usando as mesmas hipóteses de [5], finalmente Chazottes, Gambaudo e Ugalde demonstraram o mesmo resultado para uma classe mais geral de potenciais em [7] usando técnicas de renormalização.

No caso de subshifts de Markov com alfabeto enumerável, ou seja o caso não-compacto, um dos primeiros resultados na direção de limites em temperatura zero foi obtido por Jenkinson, Mauldin e Urbański em [10], eles demostraram existência de pontos de acumulação para a sequência de estados de equilíbrio $\left(\mu_{t f}\right)_{t>1}$ quando $t \rightarrow \infty$ no caso que $\Sigma$ é finitamente primitivo e o potencial tem variação somável e pressão finita, além disso demonstraram que os pontos de acumulação dessa sequência são medidas $f$-maximizantes. Finalmente Kempton demonstrou em [12] que quando o potencial é Markov, o subshift é topologicamente mixing e satisfaz a propriedade $B I P^{1}$, então existe o limite em temperatura zero.

É importante ressaltar que a condição do potencial ser localmente constante não pode ser removida, mesmo no caso compacto, para demonstrar existência do limite em temperatura zero. Chazottes e Hochman em [8] deram um exemplo de um subshift de tipo finito $\Sigma \subsetneq\{0,1\}^{\mathbb{N}}$ para o qual existe um potencial Lipschitz no qual a sequência de estados de equilíbrio diverge quando $t \rightarrow \infty$. Mais recentemente Coronel e Rivera-Letelier em [9] mostraram que no caso compacto, mais especificamente quando $\Sigma=\left(S^{1}\right)^{\mathbb{N}}$, dadas quaisquer duas medidas ergódicas $\mu^{+}$e $\mu^{-}$com a mesma entropia, sempre é possível construir um potencial Lipschitz tal que a sequência de estados

\footnotetext{
${ }^{1}$ Para subshifts topologicamente mixing esta propriedade é equivalente ao subshift ser finitamente primitivo.
} 
de equilíbrio associada ao potencial tem como pontos de acumulação duas medidas isomorfas a as medidas $\mu^{+}$e $\mu^{-}$respectivamente.

Nosso segundo resultado generaliza o resultado de Kempton em [12] para subshifts de Markov topologicamente transitivos.

Teorema B. Seja $\Sigma$ um subshift de Markov topologicamente transitivo e $f$ um potencial de Markov somável, com pressão de Gurevic finita e $V_{1}(f)<\infty$. Então a sequência de estados de equilíbrio $\left(\mu_{t f}\right)_{t>1}$ converge quando $t \rightarrow \infty$ para uma medida $\mu_{\infty} \in \mathcal{M}_{\max }(f)$.

Usando as mesmas condições de [10], Morris demonstrou em [16] existência do limite da sequência $\left(h\left(\mu_{t f}\right)\right)_{t>1}$ quando $t \rightarrow \infty$ no caso de subshifts de Markov finitamente primitivos, além disso mostrou que a sequência converge para o supremo das entropias das medidas de probabilidade $f$-maximizantes.

Nosso último resultado é uma generalização do resultado de Morris no sentido que mostra o mesmo comportamento das entropias quando $t \rightarrow \infty$, porém usamos as mesmas hipóteses do teorema 1 que são bem mais gerais desde o ponto de vista dinâmico das que são usadas em [16]. O resultado é o seguinte:

Teorema C. Seja $\Sigma$ um subshift de Markov topologicamente transitivo e $f$ um potencial somável, com pressão de Gurevic finita e $\bar{V}(f)<\infty$. Então

$$
h\left(\mu_{\infty}\right)=\lim _{t \rightarrow \infty} h\left(\mu_{t f}\right)=\sup \left\{h(\mu): \mu \in \mathcal{M}_{\max }(f)\right\}
$$

onde $\mu_{\infty}$ é ponto de acumulação da família $\left(\mu_{t f}\right)_{t>1}$.

A importância deste trabalho é que nas hipóteses dos teoremas o subshift não satisfaz a propriedade $B I P$ a qual garante existência de medidas de Gibbs [18], portanto muitas das ferramentas dadas no formalismo termodinâmico para subshifts de Markov com alfabeto enumerável não são validas neste caso.

A ideia da prova é fazer uma aproximação de nosso subshift $\Sigma$ por uma sequência crescente de subshifts compactos $\left(\Sigma_{k}\right)_{k \geq 0}$ e usando argumentos de rigidez garantir existência de pontos de acumulação família de medidas de probabilidade quando $t \rightarrow \infty$. Além disso vamos usar a existência do limite em temperatura zero no caso compacto para demonstrar a existência do limite no caso que nós trabalhamos usando um argumento muito parecido a um argumento diagonal.

As principais ferramentas que vamos usar para fazer essa aproximação são a caracterização dada por Leplaideur em [13] do limite em temperatura zero para o caso de subshifts com alfabeto 
finito e o algoritmo de renormalização demonstrado por Chazottes, Gambaudo e Ugalde em [7]. Também vamos usar o resultado demonstrado por Bissacot e Freire em [3] no qual garantem a existência de medidas maximizantes para potenciais coercivos definidos sobre subshifts de Markov topologicamente transitivos, e por último vamos usar o resultado demonstrado por Buzzi e Sarig em [6] no qual é garantida a unicidade do estado de equilíbrio quando o subshift tem alfabeto enumerável e a dinâmica é topologicamente transitiva. Para a demonstração do último resultado vamos usar que todo argumentos bastante simples da teoria da medida e alguns dos princípios variacionais que satisfaz a pressão topológica, além disso vamos usar o resultado demonstrado por Morris em [16].

O trabalho está organizado da seguinte forma:

Na primeira parte do capítulo 2 fazemos uma introdução à teoria ergódica lembrando alguns dos resultados clássicos os quais serão usados ao longo do trabalho; na segunda parte aparecem alguns resultados do formalismo termodinâmico no contexto de subshifts de Markov os quais foram demonstrados em maior parte por Jenkinson, Mauldin, Sarig e Urbański, isso na busca dos resultados que aparecem nos capítulos 3 e 5 .

Na primeira parte do capítulo 3 seguindo o trabalho do Sarig em [20] vamos definir o operador de Ruelle associado a um potencial $f$ no caso de subshifts de Markov com alfabeto enumerável, além disso estudaremos algumas das principais propriedades deste operador e de seu operador dual procurando uma caracterização dos estados de equilíbrio associados ao potencial $f$, na segunda parte do capítulo vamos estudar o resultado de Buzzi e Sarig em [6] no qual é garantida a unicidade do estado de equilíbrio associado ao potencial $f$ quando tem variação somável e pressão finita.

Na primeira parte do capítulo 4 estudaremos o resultado de Bissacot e Freire em [3] que garante existência de medidas maximizantes para subshifts de Markov topologicamente transitivos, na segunda parte do capítulo estudaremos o resultado de Leplaideur em [13] que caracteriza o limite em temperatura zero quando o subshift é compacto, e a renormalização construída por Chazottes, Gambaudo e Ugalde em [7].

No capítulo 5 aparecem os três resultados obtidos neste trabalho. Nosso primeiro resultado garante existência de um único estado de equilíbrio associado ao potencial tf para cada $t>1$. O segundo resultado é uma generalização do trabalho de Kempton em [12] para o caso de subshifts de Markov topologicamente transitivos com alfabeto enumerável, além disso usando a caracterização obtida em [13] nós obtemos uma caracterização no caso não-compacto. Nosso último resultado é uma generalização do resultado obtido por Morris em [16] para o caso subshifts de Markov topologicamente transitivos com alfabeto enumerável. 


\section{Capítulo 2}

\section{Preliminares}

A teoria ergódica tem seus origens no estudo dos fluxos Hamiltonianos, porém o H. Poincaré discretizo o problema para conseguir uma solução, isso explica porque a maioria dos resultados clássicos que vamos abordar neste capítulo são propostos para o caso de dinâmica discreta, no entanto eles podem ser generalizados na maioria dos casos com pouca dificuldade para fluxos.

Na primeira seção deste capítulo vamos ver alguns desses resultados fundamentais, entre eles o teorema de recorrência de Poincaré, o teorema ergódico de Birkhoff e o teorema de decomposição ergódica. Além disso vamos mostrar algumas das suas consequências mais importantes.

Na segunda seção do capítulo vamos dar uma definição para os subshifts de Markov e vamos estudar alguns resultados fundamentais do formalismo termodinâmico nesse contexto. Uma das coisas mais importantes que vamos fazer nesta seção vai ser definir a pressão de Gurevic, a qual é bastante útil neste contexto porque coincide com a pressão topológica ${ }^{1}$.

A maioria dos resultados que aparecem neste capítulo são demonstrados em [1], [15], [17] e [19].

\section{$2.1 \quad$ Teoria ergódica}

Para falar de teoria ergódica no contexto dos sistemas dinâmicos discretos precisamos no minimo de um espaço mensurável $(X, \mathcal{B})$ e um sistema dinâmico ${ }^{2}(T, X)$.

Nesta seção vamos estudar alguns tipos de medidas que tem uma relação direta com a dinâmica, entre elas estão as medidas não-singulares, as medidas invariantes, etc. A continuação vamos dar algumas definições que serão usadas ao longo deste trabalho.

1. Uma medida $\nu$ é absolutamente contínua com respeito à medida $\mu$, e o denotaremos como

\footnotetext{
${ }^{1}$ Esse resultado aparecerá demonstrado no capítulo 3.

${ }^{2}$ Ao longo deste trabalho sempre vamos ter uma dinâmica fixada.
} 
$\nu \ll \mu$, se para todo conjunto mensurável $E \subset X$ tal que $\mu(E)=0$, também é satisfeito $\nu(E)=0$. Usaremos a notação $\nu \sim \mu$ quando $\nu \ll \mu$ e $\mu \ll \nu$, ou seja $\mu(E)=0$ se, e somente se $\nu(E)=0$.

2. Uma medida $\mu$ é não-singular se para todo conjunto $E \subset X$ mensurável $\mu\left(T^{-1} E\right)=0$ se, e somente se $\mu(E)=0$.

3. Uma medida não-singular $\mu$ é conservativa se todo conjunto mensurável $E \subset X$ tal que $T^{-n} E \cap E=\emptyset$ para cada $n \in \mathbb{N}$, satisfaz $\mu(E)=0$.

4. Uma medida $\mu$ é invariante se todo conjunto mensurável $E \subset X$ satisfaz $\mu\left(T^{-1} E\right)=\mu(E)$.

Observe que toda medida invariante é não-singular, no entanto o reciproco não é necessariamente certo, uma pequena modificação no enunciado do teorema 2 ajuda a garantir que toda medida de probabilidade conservativa é invariante.

Vamos denotar por $\mathcal{M}(X)$ o conjunto das medidas borelianas de probabilidade sobre $X$, e denotaremos por $\mathcal{M}_{T}(X) \subset \mathcal{M}(X)$ o conjunto das medidas de probabilidade $T$-invariantes sobre $X$.

Definimos a topologia fraca* sobre o conjunto $\mathcal{M}(X)$ como a topologia gerada pela seguinte família de vizinhanças

$$
V(\mu, \mathcal{F}, \epsilon)=\left\{\nu \in \mathcal{M}(X):\left|\int f_{i} d \nu-\int f_{i} d \mu\right|<\epsilon, \forall 1 \leq i \leq m\right\}
$$

onde $\mathcal{F}=\left\{f_{1}, \ldots, f_{m}\right\}$ e cada uma das funções $f_{i}$ é contínua limitada. Não é difícil ver que a topologia fraca* é equivalente à topologia gerada pela família de vizinhanças

$$
V^{\prime}(\mu, \mathcal{B}, \epsilon)=\left\{\nu \in \mathcal{M}(X):\left|\nu\left(B_{i}\right)-\mu\left(B_{i}\right)\right|<\epsilon, \forall 1 \leq i \leq m\right\}
$$

onde $\mathcal{B}=\left\{B_{1}, \ldots, B_{m}\right\}$ e cada um dos conjuntos $B_{i}$ é um conjunto de continuidade para $\mu$, ou seja $\mu\left(\partial B_{i}\right)=0^{3}$. Em consequência temos $\lim _{n \rightarrow \infty} \mu_{n}=\mu$ se, e somente se $\lim _{n \rightarrow \infty} \mu_{n}(B)=\mu(B)$ para todo conjunto de continuidade da medida $\mu$.

Vamos dar uma caracterização da topologia fraca* a qual vai facilitar algumas das demonstrações que apareceram de aqui para o frente.

Proposição 1. Uma sequência $\left(\mu_{n}\right)_{n \in \mathbb{N}}$ converge na topologia fraca* ${ }^{*}$ para $\mu$ se, e somente se, para

\footnotetext{
${ }^{3}$ Onde $\partial B_{i}$ é a fronteira topológica do conjunto $B_{i}$.
} 
toda função continua limitada $g: X \rightarrow \mathbb{R}$ temos

$$
\lim _{n \rightarrow \infty} \int g d \mu_{n}=\int g d \mu
$$

De aqui para o frente sempre que falemos de convergência de medidas de probabilidade, estaremos falando no contexto da topologia fraca*.

Além disso, é possível demonstrar que quando o espaço $X$ é métrico separável, então $\mathcal{M}(X)$ também é espaço métrico separável dotado com a topologia fraca*, além disso quando $X$ compacto temos que $\mathcal{M}(X)$ também é compacto.

No caso que o espaço $X$ não é compacto dizemos que um subconjunto $\mathcal{K} \subset \mathcal{M}(X)$ é justo, se para todo $\epsilon>0$ existe um conjunto compacto $K \subset X$ tal que $\mu\left(K^{c}\right)<\epsilon$ para toda $\mu \in \mathcal{K}$.

A definição de conjunto justo é bastante útil, porque as medidas pertencentes a um conjunto justo tem seu suporte contido quase por completo num conjunto compacto o qual vai facilitar muitas contas no caso não compacto.

O seguinte teorema é bastante útil na busca de garantir existência de pontos de acumulação na topologia fraca* para uma sequência arbitraria de medidas usando a definição de conjunto justo.

Teorema 1 (Prohorov). [2] Seja $(X, \mathcal{B})$ um espaço métrico, separável e completo. O conjunto $\mathcal{K} \subset \mathcal{M}(X)$ é justo se, e somente se toda sequência em $\mathcal{K}$ possui alguma subsequência convergente.

Um dos objetivos principais da teoria ergódica é o estudo das órbitas tipicas do sistema para quase todo ponto, nessa direção um dos primeiros resultados importantes desta área foi demonstrado por Poincaré no siglo XIX e fala do comportamento recorrente das orbitas típicas de quase todo ponto num conjunto de medida positiva, esse teorema foi uma consequência da discretização da dinâmica dos fluxos Hamiltonianos definidos sobre espaços euclídeos.

Teorema 2 (Recorrência de Poincaré). [17] Seja $\mu \in \mathcal{M}_{T}(X)$ e $E \subset X$ mensurável com $\mu(E)>0$. Então para $\mu$-quase todo ponto $x \in E$ existem infinitos valores de $n$ para os quais $T^{n} x \in E$

Um conjunto $E \subset X$ é chamado invariante se $T^{-1} E=E$, e uma medida não-singular $\mu$ é chamada ergódica se para todo conjunto mensurável invariante $E \subset X$ é satisfeito $\mu(E)=0$ ou $\mu\left(E^{c}\right)=0$, de aqui para o frente vamos denotar o conjunto das medidas de probabilidade ergódicas por $\mathcal{M}_{\text {erg }}(X)$, claramente $\mathcal{M}_{\text {erg }}(X) \subset \mathcal{M}_{T}(X)$, além disso é fácil dar conta que o conjunto $\mathcal{M}_{T}(X)$ é um simplex e que o conjunto dos seus pontos extremais é $\mathcal{M}_{\text {erg }}(X)$.

Dizemos que uma função integrável $f$ é invariante se satisfaz $f \circ T=f$. A continuação vamos dar uma caracterização das medidas de probabilidade ergódicas usando funções invariantes. 
Lema 1. [17] Uma medida $\mu \in \mathcal{M}_{T}(X)$ é ergódica se, e somente se toda função invariante é constante $\mu$-quase sempre.

Para $f: X \rightarrow \mathbb{R}$ mensurável e $n \geq 1$ vamos denotar à $n$-ésima suma ergódica da $f$ por $S_{n} f(x)=\sum_{i=0}^{n-1} f\left(T^{i} x\right)$ e dado um ponto arbitrário $x \in X$ definimos sua media ergódica como $\lim _{n \rightarrow \infty} \frac{1}{n} S_{n} f(x)$, observe que para o caso $x \in \operatorname{Per}(\Sigma)$ o limite existe, porém em casos mais gerais não é necessariamente certo.

O teorema ergódico de Birkhoff é um dos resultados clássicos da teoria ergódica, ele garante existência das medias ergódicas para quase todo ponto, além disso o teorema dá condições para que as medias ergódicas coincidam com as medias espaciais, esta propriedade é conhecida como hipótese ergódica.

Teorema 3 (Ergódico de Birkhoff). [17] Seja $\mu \in \mathcal{M}_{T}(X)$ e $f \in L^{1}(\mu)$, então o limite

$$
f^{*}(x)=\lim _{n \rightarrow \infty} \frac{1}{n} S_{n} f(x)
$$

existe para $\mu$-quase todo ponto $x \in X$, além disso $\int f^{*} d \mu=\int f d \mu$. Em particular se a medida de probabilidade $\mu$ é ergódica, então $f^{*}(x)=\int f d \mu$ para $\mu$-quase todo ponto $x \in X$.

Observe que se a medida de probabilidade $\mu$ é ergódica, ainda que $\int f d \mu=\infty$ a conclusão do teorema ergódico de Birkhoff continua verdadeira.

Com efeito para qualquer $M>0$ temos que $\left(f 1_{[f<M]}\right)^{*}(x)=\int f 1_{[f<M]} d \mu$, e para cada $n \in \mathbb{N}$ temos $\frac{1}{n} S_{n} f(x) \geq \frac{1}{n} S_{n}\left(f 1_{[f<M]}\right)(x)$, então

$$
\liminf _{n \rightarrow \infty} \frac{1}{n} S_{n} f(x) \geq \int f 1_{[f<M]} d \mu
$$

Portanto dado que $M$ é arbitrário temos que $\liminf _{n \rightarrow \infty} \frac{1}{n} S_{n} f(x)=\infty$, do qual segue imediatamente que $f^{*}(x)=\infty$.

Seja $(X, \mathcal{B}, \mu)$ um espaço de probabilidade, uma família de subconjuntos enumeráveis $\mathcal{P}$ é uma partição mensurável de $X$ se os seus elementos são disjuntos dois a dois, exceto possivelmente num conjunto de medida zero, e além disso a união de todos os elementos de $\mathcal{P}$ tem medida total. De aqui para o frente sempre que falemos de partição estaremos falando de uma partição mensurável.

Seja $\pi: X \rightarrow \mathcal{P}$ a projeção natural, ou seja a função $x \mapsto \mathcal{P}(x)$ onde $\mathcal{P}(x) \in \mathcal{P}$ é o elemento da partição que contém o ponto $x$, dizemos que $\mathcal{Q} \subset \mathcal{P}$ é mensurável se, e somente se $\pi^{-1} \mathcal{Q}$ é mensurável. 
Para todo conjunto $\mathcal{Q} \subset \mathcal{P}$ mensurável definimos a sua medida como

$$
\hat{\mu}(\mathcal{Q})=\mu\left(\pi^{-1} \mathcal{Q}\right)
$$

a medida $\hat{\mu}$ é conhecida como medida quociente.

O seguinte teorema faz uma decomposição dos elementos do $\operatorname{simplex} \mathcal{M}_{T}(X)$ como combinação convexa de pontos extremais que são elementos de $\mathcal{M}_{\text {erg }}(X)$.

Teorema 4 (Decomposição ergódica). Seja $X$ um espaço completo separável e $\mu \in \mathcal{M}_{T}(X)$. Então existe uma partição $\mathcal{P}$ de $X$ e uma família de medidas de probabilidade $\left(\mu_{P}\right)_{P \in \mathcal{P}}$ em $X$, tal que

1. $\mu_{P}(P)=1$ para $\hat{\mu}$-quase todo $P \in \mathcal{P}$;

2. $P \mapsto \mu_{P}(E)$ é mensurável, para todo $E \subset X$ mensurável;

3. $\mu_{P}$ é ergódica para $\hat{\mu}$-quase todo $P \in \mathcal{P}$;

4. $\int f d \mu=\int\left(\int f d \mu_{P}\right) d \hat{\mu}(P)$ para toda $f: X \rightarrow \mathbb{R}$ mensurável limitada.

Vamos denotar como $\mu=\int \mu_{P} d \hat{\mu}(P)$ a decomposição ergódica da medida $\mu$.

Dada uma medida $\mu \in \mathcal{M}(X)$ e uma partição $\mathcal{P}$, definimos a entropia da $\mu$ associada à partição $\mathcal{P}$ como

$$
H(\mu \mid \mathcal{P}):=\sum_{P \in \mathcal{P}}-\mu(P) \log (\mu(P))
$$

Uma partição $\mathcal{P}$ é mais fina que a partição $\mathcal{Q}$, se para todo $Q \in \mathcal{Q}$ existe $P \in \mathcal{P}$ tal que $P \subset Q$, neste caso vamos usar a notação $\mathcal{Q} \prec \mathcal{P}$.

Observe que quando $\mu \in \mathcal{M}_{T}(X)$ temos $H(\mu \mid \mathcal{P})=H\left(\mu \mid T^{-1}(\mathcal{P})\right)$, além disso quando $\mathcal{Q} \prec \mathcal{P}$ temos $H(\mu \mid \mathcal{Q}) \leq H(\mu \mid \mathcal{P})$.

Dada uma partição $\mathcal{P}$ de $X$ vamos denotar $\mathcal{P}^{n}=\bigvee_{j=0}^{n-1} T^{-j} \mathcal{P}$. Observe que se $m<n$, então $\mathcal{P}^{m} \prec \mathcal{P}^{n}$, além disso para $\mu \in \mathcal{M}_{T}(X)$ é satisfeito $H\left(\mu \mid \mathcal{P}^{n+m}\right) \leq H\left(\mu \mid \mathcal{P}^{n}\right)+H\left(\mu \mid \mathcal{P}^{m}\right)$, ou seja a sequência $\left(H\left(\mu \mid \mathcal{P}^{n}\right)\right)_{n \geq 1}$ é subaditiva sempre que $\mu \in \mathcal{M}_{T}(X)$.

Dada uma uma medida $\mu \in \mathcal{M}_{T}(X)$ e uma partição $\mathcal{P}$ de $X$, definimos a entropia de $T$ com respeito à medida $\mu$ e à partição $\mathcal{P}$ como

$$
h(\mu, \mathcal{P}):=\lim _{n \rightarrow \infty} \frac{1}{n} H\left(\mu \mid \mathcal{P}^{n}\right)=\inf _{n \geq 1} \frac{1}{n} H\left(\mu \mid \mathcal{P}^{n}\right) .
$$

O limite em (2.1) sempre existe pela subaditividade da sequência $\left(H\left(\mu \mid \mathcal{P}^{n}\right)\right)_{n \geq 1}$, além disso é imediato que para quaisquer duas partições $\mathcal{Q} \prec \mathcal{P}$ é satisfeito $h(\mu, \mathcal{Q}) \leq h(\mu, \mathcal{P})$, mais ainda como 
$\bigvee_{j=0}^{n-1} T^{-j} \mathcal{P}=\mathcal{P}^{n+k-1}$ segue imediatamente que $h(\mu, \mathcal{P})=h\left(\mu, \mathcal{P}^{n}\right)$ para todo $n \geq 0$. Definimos a entropia de Kolmogorov-Sinai da medida $\mu$ como

$$
h(\mu):=\sup _{\mathcal{P}} h(\mu, \mathcal{P})
$$

onde o supremo é tomado sobre todas as partições $\mathcal{P}$ de $X^{4}$.

Lema 2. [17] Seja $\mathcal{P}_{1} \prec \ldots \prec \mathcal{P}_{n} \prec \ldots$ uma sequência não decrescente de partições tal que $\bigcup_{n \geq 1} \mathcal{P}_{n}$ gera a $\sigma$-álgebra dos conjuntos mensuráveis a menos de medida nula, então $h(\mu)=\lim _{n \rightarrow \infty} h\left(\mu, \mathcal{P}_{n}\right)$

Observe que se $\bigcup_{n \geq 1} \mathcal{P}^{n}$ gera a $\sigma$-álgebra dos conjuntos mensuráveis, então $h(\mu)=h(\mu, \mathcal{P})$.

Finalmente dizemos que a dinâmica $T: X \rightarrow X$ é topologicamente transitiva se para quaisquer par de conjuntos abertos $U, V \subset X$ existe $n \in \mathbb{N}$ tal que $T^{-n} U \cap V \neq \emptyset$, e dizemos que a dinâmica $T: X \rightarrow X$ é topologicamente mixing se para quaisquer par de conjuntos abertos $U, V \subset X$ existe $N \in \mathbb{N}$ tal que $T^{-n} U \cap V \neq \emptyset$ para todo $n \geq N$.

\subsection{Formalismo termodinâmico}

Seja $\mathcal{A} \subset \mathbb{R}$ um alfabeto enumerável ${ }^{5}$ e $\mathbf{M}$ uma matriz de 0 's e 1's indexada em $\mathcal{A} \times \mathcal{A}$ tal que nenhuma das suas filas ou colunas está composta unicamente por 0's. Definimos o subshift de Markov $(\sigma, \Sigma)$ com alfabeto $\mathcal{A}$ e matriz de incidência $\mathbf{M}$ como

$$
\Sigma=\left\{x \in \mathcal{A}^{\mathbb{N}}: \mathbf{M}_{x_{i}, x_{i+1}}=1\right\}
$$

junto com a transformação $\sigma: \Sigma \rightarrow \Sigma$ sendo o shift a esquerda, ou seja $\sigma\left(\left(x_{n}\right)_{n \in \mathbb{N}}\right)=\left(x_{n+1}\right)_{n \in \mathbb{N}}$.

De aqui para o frente vamos supor que $(\sigma, \Sigma)$ é topologicamente transitivo.

O formalismo termodinâmico estuda existência e propriedades das medidas $\sigma$-invariantes que maximizam $P_{\mu}(f):=h(\mu)+\int f d \mu$, essas medidas são chamadas de estados de equilíbrio.

Nesta seção também vamos definir a pressão de Gurevic $P_{G}(f)$ a qual é bastante útil neste contexto, além disso no capítulo 3 vamos mostrar que no caso de subshifts de Markov topologicamente transitivos com alfabeto enumerável, sempre que a pressão seja finita essa definição coincide com a pressão topológica

$$
P_{t o p}(f):=\sup \left\{P_{\mu}(f): \int f d \mu>-\infty\right\}
$$

\footnotetext{
${ }^{4}$ Observe que esta definição depende do sistema $(T, X)$.

${ }^{5}$ Para fazer os cálculos mais simples vamos supor $\mathcal{A}=\mathbb{N}$, porém a definição faz sentido em conjuntos não enumeráveis.
} 
Dada uma palavra $\omega=a_{0} \ldots a_{n-1}$ definimos o cilindro $[\omega]=\left\{x \in \Sigma: x_{0} \ldots x_{n-1}=a_{0} \ldots a_{n-1}\right\}$, dado $\theta \in(0,1)$ e $t(x, y)=\inf \left\{n \in \mathbb{N}: x_{0} \ldots x_{n-1} \neq y_{0} \ldots y_{n-1}\right\}$ definimos a métrica $d_{\theta}$ sobre $\Sigma$ como $d_{\theta}(x, y)=\theta^{t(x, y)}$. Além disso é fácil demonstrar que para quaisquer $\theta \in(0,1)$ a topologia gerada pela métrica $d_{\theta}$ coincide com a topologia gerada pelos cilindros, portanto $\Sigma$ é um espaço métrico separável.

Seja $f: \Sigma \rightarrow \mathbb{R}$ um potencial ${ }^{6}$, dizemos que $f$ tem variação somável se

$$
V(f):=\sum_{n \geq 2} V_{n}(f)<\infty
$$

além disso $V(f)<\infty$ implica $\sup _{n \geq 1} V_{n+1}\left(S_{n} f\right)<\infty$.

O potencial $f$ é coercivo se

$$
\lim _{a \rightarrow \infty}\left(\left.\sup f\right|_{[a]}\right)=-\infty
$$

e chamaremos o potencial $f$ somável se satisfaz

$$
\sum_{a \in \mathcal{A}} \exp \left(\sup \left(\left.f\right|_{[a]}\right)\right)<\infty
$$

Observe que todo potencial somável é coercivo mas a recíproca em geral não é valida. A propriedade do potencial ser somável vai ser muito importante na demonstração da existência dos estados de equilíbrio o qual é um dos resultados mais relevantes deste trabalho.

Lema 3. [16] Se o potencial $f$ é somável, então para todo $t>1$

$$
\sum_{a \in \mathcal{A}} \sup \left(-\left.t f\right|_{[a]}\right) \exp \left(\sup \left(\left.t f\right|_{[a]}\right)\right)<\infty
$$

Dados dois potenciais $f, f^{\prime}$ e uma constante $c$, dizemos que o potencial $f^{\prime}$ é cohomologo ao potencial $f+c$ via a função $\varphi$ se é satisfeito $f^{\prime}=f+\varphi-\varphi \circ \sigma+c$, o qual denotaremos como $f^{\prime} \backsim f+c$, no caso $c=0$ dizemos que os potenciais são cohomologos.

Esta propriedade é bastante interessante no sentido que potenciais cohomologos conservam muitas características, por exemplo tem os mesmos estados de equilíbrio, tem as mesmas medidas maximizantes e a mesma pressão de Gurevic. O seguinte teorema dá uma relação entre as somas ergódicas das órbitas periódicas e a cohomologia o qual é bastante útil em algumas das provas que apareceram de aqui para o frente.

\footnotetext{
${ }^{6}$ Vamos entender potencial como uma função continua.
} 
Teorema 5 (Livsic). [19] Sejam $f, f^{\prime}$ potenciais com $V(f), V\left(f^{\prime}\right)<\infty$. Então o $f^{\prime} \backsim f$ via uma função $\varphi$ se, e somente se $S_{n} f(x)=S_{n} f^{\prime}(x)$ para cada $x \in \Sigma$ tal que $\sigma^{n} x=x$.

Para cada $a \in \mathcal{A}$ definimos

$$
Z_{n}(f, a):=\sum_{\sigma^{n} x=x} \exp \left(S_{n} f(x)\right) 1_{[a]}(x)
$$

Lema 4. [19] Seja $f$ um potencial com $\sup _{n \geq 1} V_{n+1}\left(S_{n} f\right)<\infty$, então para quaisquer $a, b \in \mathcal{A}$ existem constantes $C_{1}, C_{2}>0$ e $k_{1}, k_{2} \in \mathbb{N}$ tais que

$$
C_{1} Z_{n-k_{1}}(f, a) \leq Z_{n}(f, b) \leq C_{2} Z_{n+k_{2}}(f, a)
$$

para cada $n \geq k_{1}$

Dado $a \in \mathcal{A}$ definimos a pressão de Gurevic do potencial $f$ como

$$
P_{G}(f):=\lim _{n \rightarrow \infty} \frac{1}{n} \log Z_{n}(f, a) .
$$

Segue do lema 4 que para todo potencial com $\sup _{n \geq 1} V_{n+1}\left(S_{n} f\right)<\infty$ a definição de pressão de Gurevic é independente do $a \in \mathcal{A}$ escolhido ${ }^{7}$.

Lema 5. (Propriedades da pressão de Gurevic)[19]

i) $P_{G}(f)>-\infty$.

ii) $P_{G}(f+c)=P_{G}(f)+c$.

iii) Para todo $t \in[0,1], P_{G}\left(t f+(1-t) f^{\prime}\right) \leq t P_{G}(f)+(1-t) P_{G}\left(f^{\prime}\right)$.

iv) Se $f^{\prime} \backsim f+c$, então $P_{G}\left(f^{\prime}\right)=P_{G}(f)+c$.

Lema 6. [20]

$$
P_{G}(f)=\sup \left\{P_{G}\left(\left.f\right|_{\Sigma^{\prime}}\right): \Sigma^{\prime} \subset \Sigma e ́ \text { subshift compacto }\right\}
$$

Fazendo um procedimento similar à demostração feita em [20] é possível demonstrar que para uma sequência estritamente crescente de subshifts compactos $\left(\Sigma_{k}\right)_{k \in \mathbb{N}}$ é satisfeito

$$
P_{G}(f)=\sup \left\{P_{G}\left(\left.f\right|_{\Sigma_{k}}\right): k \in \mathbb{N}\right\},
$$

\footnotetext{
${ }^{7}$ Sempre que falemos de $P_{G}(f)$, estaremos supondo que $\sup _{n \geq 1} V_{n+1}\left(S_{n} f\right)<\infty$.
} 
isso porque para todo subshift compacto $\Sigma^{\prime}$ existe $k_{0} \in \mathbb{N}$ tal que $\Sigma^{\prime} \subset \Sigma_{0}^{8}$.

${ }^{8}$ Este resultado vai ser necessário no capítulo 5. 


\section{Capítulo 3}

\section{Operador de Ruelle e estados de}

\section{equilíbrio}

Neste capítulo vamos a definir o operador de Ruelle associado ao potencial $f$ no caso de subshifts de Markov topologicamente transitivos com alfabeto enumerável, é importante ressaltar que o conjunto de funções nas quais o operador está bem definido é bem mais difícil de caracterizar que no caso compacto, embora no caso não-compacto é possível dar condições sobre o $f$ na busca que o operador fique bem definido sobre o conjunto de funções integráveis com respeito à automedida associada a seu operador dual [19], além disso vamos mostrar algumas propriedades deste operador e usaremos ele na demonstração da unicidade dos estados de equilíbrio.

De aqui para o frente vamos usar a notação $A=B^{ \pm 1}$ quando $B^{-1} \leq A \leq B$, e $A=B \pm C$ quando $B-C \leq A \leq B+C$, onde $A, B, C>0$.

\subsection{Operador de Ruelle}

Definimos o operador de Ruelle associado ao potencial $f$ como

$$
\left(L_{f} g\right)(x):=\sum_{\sigma y=x} \exp (f(y)) g(y)
$$

é claro que no caso geral o operador não está bem definido porque a soma pode ser divergente, porém no caso que o potencial $f$ é positivamente recorrente em [20] dão condições para garantir que o operador $L_{f}$ converge sobre $L^{1}(\nu)$, onde $\nu$ é a única automedida do operador dual, ou seja é 
a única medida que satisfaz $L_{f}^{*} \nu=\lambda \nu, \operatorname{com} \lambda=\exp \left(P_{G}(f)\right)$ e $L_{f}^{*}$ definido por

$$
\int g d\left(L_{f}^{*} \mu\right):=\int L_{f} g d \mu
$$

Dizemos que uma medida $\mu$ é uma medida $R P F$ associada ao potencial $f$ se é da forma $\mu=h d \nu$ onde $h$ positiva continua, $L_{f} h=\lambda h, \int h d \nu=1$, e $\nu, \lambda$ são definidos como no paragrafo anterior.

Para uma medida não-singular $\mu$ e cada $E \subset \Sigma$ mensurável $^{1}$, definimos a medida $\mu \circ \sigma$ como

$$
\mu \circ \sigma(E):=\sum_{a \in \mathcal{A}} \mu(\sigma(E \cap[a]))
$$

Observe que $\mu \ll \mu \circ \sigma$, portanto a função $\frac{d \mu}{d \mu \circ \sigma}$ está bem definida, além disso para cada $g: \Sigma \rightarrow \mathbb{R}$ mensurável

$$
\int g(x) d(\mu \circ \sigma)(x)=\sum_{a \in \mathcal{A}} \int g(a x) 1_{\sigma[a]}(x) d \mu(x)
$$

Lema 7. [20] A medida $\nu$ satisfaz $L_{f}^{*} \nu=\lambda \nu$ se, e somente se

$$
\frac{d \nu}{d \nu \circ \sigma}=\lambda^{-1} \exp (f)
$$

Seja $\Sigma$ um subshift de Markov e $\alpha=\{[a]: a \in \mathcal{A}\}$ a sua partição natural. Vamos lembrar duas formulas bastante conhecidas na teoria ergódica que vão ser necessárias em algumas das demonstrações que aparecem de aqui para o frente.

Formula de Rokhlin: Seja $\mu \in \mathcal{M}_{\sigma}(\Sigma)$ tal que $H\left(\mu \mid \alpha^{n}\right)<\infty$ para cada $n \geq 1$, então

$$
h(\mu)=-\int \log \left(\frac{d \mu}{d \mu \circ \sigma}\right) d \mu .
$$

Formula de Kâc: Para $\mu \in \mathcal{M}_{\sigma}(\Sigma)$, cada $f \in L^{1}(\mu)$ e todo conjunto mensurável $E \subset \Sigma$ com $\mu(E)>0$ é satisfeito

$$
\int 1_{E}(x)\left(\sum_{j=0}^{\varphi_{E}(x)-1} f\left(\sigma^{j} x\right)\right) d \mu(x)=\int f(x) d \mu(x),
$$

onde $\varphi_{E}(x)=1_{E}(x) \inf \left\{n \geq 1: \sigma^{n} x \in E\right\}$.

É importante ressaltar que a formula de Rokhlin sempre é verdade no caso compacto, embora no caso não-compacto não é necessariamente certa porque é possível que exista algum $n^{\prime}$ tal que

\footnotetext{
${ }^{1}$ Em geral $\mu \circ \sigma(E) \neq \mu(\sigma(E))$.
} 
$H\left(\mu \mid \alpha^{n^{\prime}}\right)=\infty$, no entanto a formula de Kâc sempre é verdadeira.

Lema 8. [19] Seja $\Sigma$ um subshift de Markov topologicamente transitivo, $f: \Sigma \rightarrow \mathbb{R} \operatorname{com} V(f)<\infty$ e $\nu$ uma medida invariante finita sobre cilindros tal que $L_{f}^{*} \nu=\nu$, então $\nu$ é ergódica.

Demonstração. Sejam $\underline{a}=a_{0} \ldots a_{n-1}$ e $\underline{b}=b_{0} \ldots b_{n-1}$ tais que $\underline{a} \cap \sigma^{-n} \underline{b}$ é admissível, e seja $M=\exp \left(\sup _{n \geq 1} V_{n+1}\left(S_{n} f\right)\right)$.

Afirmação 1. Podemos supor que $\nu(\Sigma)=1$.

Com efeito, seja $\left(\xi_{a}\right)_{a \in \mathcal{A}}$ uma sequência de números reais positivos tais que a função $\gamma=$ $\sum_{a \in \mathcal{A}} \xi_{a} 1_{[a]}$ satisfaz $\int \gamma d \nu=1$. Definimos a medida $\tilde{\nu}=\gamma d \nu$, observe que $\tilde{\nu}(\Sigma)=1$, além disso como $\gamma>0$, então $\tilde{\nu} \backsim \nu$, portanto $\tilde{\nu}$ ergódica se, e somente se, $\nu$ ergódica.

Definimos $\tilde{f}=\log \left(\frac{d \tilde{\nu}}{d \tilde{\nu} \circ \sigma}\right)$, portanto segue do lema 7 que $L_{\tilde{f}}^{*} \tilde{\nu}=\tilde{\nu}$ e

$$
\log \left(\frac{d \tilde{\nu}}{d \tilde{\nu} \circ \sigma}\right)=\log \left(\frac{d \nu}{d \nu \circ \sigma}\right)+\log \gamma-\log \gamma \circ \sigma=f+\log \gamma-\log \gamma \circ \sigma
$$

além disso dado que $V_{k}(\log \gamma-\log \gamma \circ \sigma)=0$ para todo $k \geq 2$, então $V(\tilde{f}) \leq V(f)<\infty$.

Afirmação 2. $\exp \left(S_{n} f(\underline{a} x)\right)=M^{ \pm 1} \frac{\nu[\underline{a}]}{\nu\left(\sigma\left[a_{n-1}\right]\right)}$.

Com efeito, dado que para todo $x, y \in \Sigma$ temos $\exp \left(S_{n} f(\underline{a} x)\right)=M^{ \pm 1} \exp \left(S_{n} f(\underline{a} y)\right)$, então

$$
\begin{aligned}
\exp \left(S_{n} f(\underline{a} x)\right) & =\frac{1}{\nu\left(\sigma\left[a_{n-1}\right]\right)} \int \exp \left(S_{n} f(\underline{a} x)\right) 1_{\sigma\left[a_{n-1}\right]}(y) d \nu(y) \\
& =\frac{M^{ \pm 1}}{\nu\left(\sigma\left[a_{n-1}\right]\right)} \int \exp \left(S_{n} f(\underline{a} y)\right) 1_{\sigma\left[a_{n-1}\right]}(y) d \nu(y) \\
& =\frac{M^{ \pm 1}}{\nu\left(\sigma\left[a_{n-1}\right]\right)} \int L_{f}^{n} 1_{[\underline{a}]}(y) d \nu(y) \\
& =M^{ \pm 1} \frac{\nu[\underline{a}]}{\nu\left(\sigma\left[a_{n-1}\right]\right)}
\end{aligned}
$$

Afirmação 3. $\frac{\nu\left[\underline{a} \cap \sigma^{-n} \underline{b}\right]}{\nu[\underline{a}] \nu[\underline{b}]}=M^{ \pm 1} \frac{1}{\nu\left(\sigma\left[a_{n-1}\right]\right)}$. 
Com efeito, dado que $[\underline{b}] \cap \sigma\left[a_{n-1}\right]=[\underline{b}]$, pela afirmação 2 temos

$$
\begin{aligned}
\nu\left[\underline{a} \cap \sigma^{-n} \underline{b}\right] & =\int L_{f}^{n} 1_{\left[\underline{a} \cap \sigma^{-n} \underline{b}\right]}(x) d \nu(x) \\
& =\int \exp \left(S_{n} f(\underline{a} x)\right) 1_{\left[\sigma^{-n} \underline{b}\right]}(\underline{a} x) 1_{\sigma\left[a_{n-1}\right]}(x) d \nu(x) \\
& =\int \exp \left(S_{n} f(\underline{a} x)\right) 1_{[\underline{b}]}(x) 1_{\sigma\left[a_{n-1}\right]}(x) d \nu(x) \\
& =\int \exp \left(S_{n} f(\underline{a} x)\right) 1_{[\underline{b}]}(x) d \nu(x) \\
& =M^{ \pm 1} \frac{\nu[\underline{a}] \nu[\underline{b}]}{\nu\left(\sigma\left[a_{n-1}\right]\right)} .
\end{aligned}
$$

Pela afirmação 3, e usando novamente que $[\underline{b}]=[\underline{b}] \cap \sigma\left[a_{n-1}\right]$ concluímos que para todo conjunto $E \subset \Sigma$ mensurável

$$
\nu\left(\sigma^{-n} E \mid[\underline{a}]\right)=M^{ \pm 1} \nu\left(E \mid \sigma\left[a_{n-1}\right]\right) .
$$

Seja $E \subset \Sigma$ mensurável $\sigma$-invariante $\operatorname{com} \nu(E)>0$, e $\alpha_{n}$ a $\sigma$-álgebra gerada pelos cilindros de tamanho $n$. Para $g: \Sigma \rightarrow \mathbb{R}$ mensurável definimos a esperança condicional da $g$ dado o cilindro $\left[y_{0} \ldots y_{n-1}\right]$ como

$$
\mathbb{E}_{\nu}\left(g \mid\left[y_{0} \ldots y_{n-1}\right]\right):=\frac{\int g 1_{\left[y_{0} \ldots y_{n-1}\right]} d \nu}{\nu\left[y_{0} \ldots y_{n-1}\right]}
$$

e definimos a esperança condicional da $g$ com respeito à $\sigma$-álgebra $\alpha_{n}$ como

$$
\mathbb{E}_{\nu}\left(g \mid \alpha_{n}\right)(x):=\sum_{\left[y_{0} \ldots y_{n-1}\right] \neq \emptyset} \mathbb{E}_{\nu}\left(g \mid\left[y_{0} \ldots y_{n-1}\right]\right) 1_{\left[y_{0} \ldots y_{n-1}\right](x)}
$$

Como temos $\sigma^{-n} E=E$ para cada $n \in \mathbb{N}$, então segue que

$$
\mathbb{E}_{\nu}\left(1_{E} \mid \alpha_{n}\right)(x)=\mathbb{E}_{\nu}\left(1_{E} \mid\left[y_{0}, \ldots, y_{n-1}\right]\right)(x)=\nu\left(\sigma^{-n} E \mid\left[x_{0} \ldots x_{n-1}\right]\right),
$$

portanto usando (3.4) concluímos que $\mathbb{E}_{\nu}\left(1_{E} \mid \alpha_{n}\right)(x)=M^{ \pm 1} \nu\left(E \mid \sigma\left[x_{n-1}\right]\right)$.

Seja $a \in \mathcal{A}$ tal que $\nu(E \cap \sigma[a])>0$, então

$$
\nu[a]=\int L_{f} 1_{[a]} d \nu=\int \exp (f(a x)) 1_{\sigma[a]}(x) d \nu(x) \geq M^{-1} C \nu(\sigma[a])=M^{-1} C \nu(E \cap \sigma[a])>0 .
$$

Dado que $\nu$ é conservativa, então para $\nu$-quase todo $x \in[a]$, existem infinitos valores de $n$ para os quais $x_{n-1}=a$, portanto $\nu\left(E \mid \sigma\left[x_{n-1}\right]\right)=\nu(E \mid \sigma[a])$ quase sempre sobre $[a]$, então

$$
\limsup _{n \rightarrow \infty} \mathbb{E}_{\nu}\left(1_{E} \mid \alpha_{n}\right)(x) \geq M^{-1} \nu(E \mid \sigma[a])>0 .
$$


Além disso pelo teorema de convergência de Martingale $\limsup _{n \rightarrow \infty} \mathbb{E}_{\nu}\left(1_{E} \mid \alpha_{n}\right)(x)=1_{E}(x)$, portanto $1_{E}>0$ quase sempre sobre $[a]$, ou seja existe $E_{a} \supset E$ satisfazendo $\nu\left(E_{a}\right)=\nu(E)$ e $E_{a} \supset[a]$, portanto $\nu(E \cap[a])=\nu[a]$.

Seja $b \in \mathcal{A}$ tal que $[a] \subset \sigma[b]$, então $\nu(E \cap \sigma[b]) \geq \nu(E \cap[a])=\nu[a]>0$, pelo mesmo argumento usado acima temos que existe $E_{b} \supset E$ satisfazendo $\nu\left(E_{b}\right)=\nu(E)$ e $E_{b} \supset[b]$. Além disso se $b \in \mathcal{A}$ é tal que $[a] \subset \sigma^{n}[b]$ para algum $n \geq 1$, então existe $E_{b}^{n} \supset E$ satisfazendo $\nu\left(E_{b}^{n}\right)=\nu(E) \mathrm{e}$ $E_{b}^{n} \supset \sigma^{n-1}[b]$, portanto $E_{b}^{\prime}=\sigma^{-(n-1)} E_{b}^{n} \supset[b]$ além disso $E_{b}^{\prime} \supset E$ e $\nu\left(E_{b}^{\prime}\right)=\nu(E)$. Dado que $\Sigma$ é topologicamente transitivo, para todo $b \in \mathcal{A}$ existe $N_{a b}$ tal que $[a] \subset \sigma^{n}[b]$ para cada $n \geq N_{a b}$, portanto $E^{\prime}=\bigcap_{b \in \mathcal{A}} E_{b}^{\prime}$ satisfaz $E^{\prime} \supset E, \nu\left(E^{\prime}\right)=\nu(E)$ e $E^{\prime} \supset[b]$ para cada $b \in \mathcal{A}$, ou seja $E^{\prime}=\Sigma$.

O seguinte lema mostra que no contexto de subshifts de Markov topologicamente transitivos com alfabeto enumerável a pressão de Gurevic coincide com a pressão topológica o qual facilita muito o estudo do formalismo termodinâmico quando o alfabeto enumerável. O resultado foi demonstrado por Sarig.

Lema 9. [19] $P_{G}(f)$ satisfaz o principio variacional, ou seja

$$
P_{G}(f)=\sup \left\{P_{\mu}(f): \int f d \mu>-\infty\right\}=P_{t o p}(f)
$$

Demonstração. $(\leq)$ Seja $\epsilon>0$, pelo lema 6 existe um subshift compacto $\Sigma^{\prime} \subset \Sigma$ tal que $f^{\prime}=\left.f\right|_{\Sigma^{\prime}}$ satisfaz $P_{G}(f) \leq P_{G}\left(f^{\prime}\right)+\epsilon$. Dado que $\Sigma^{\prime}$ é compacto, então pelo teorema de Perron-Frobenious temos que existem $h: \Sigma^{\prime} \rightarrow \mathbb{R}$ positiva continua e $\nu$ medida de probabilidade satisfazendo $L_{f^{\prime}} h=$ $\lambda^{\prime} h, L_{f^{\prime}}^{*} \nu=\lambda^{\prime} \nu$ e $\nu(h)=1$, onde $\lambda^{\prime}=e^{P_{G}\left(f^{\prime}\right)}$. Seja $\mu=h d \nu$, então para todo cilindro $[\omega]=$ $\left[a_{0} \ldots a_{n-1}\right]$

$$
\begin{aligned}
\mu\left(\sigma^{-1}[\omega]\right) & =\int\left(1_{[\omega]} \circ \sigma\right) h d \nu \\
& =\int \lambda^{\prime} 1_{[\omega]} L_{f^{\prime}} h d \nu \\
& =\int 1_{[\omega]} h d \nu \\
& =\mu[\omega]
\end{aligned}
$$

ou seja $\mu$ é $\sigma$-invariante. Seja $\alpha_{\Sigma^{\prime}}=\left\{[a] \cap \Sigma^{\prime}: a \in \mathcal{A}\right\}$ uma partição de $\Sigma^{\prime}$, como $H\left(\mu \mid \alpha_{\Sigma^{\prime}}\right)<\infty$ 
e $\log h$ é continua limitada, pela formula de Rokhlin temos

$$
\begin{aligned}
h(\mu) & =-\int\left(\log \frac{d \mu}{d \mu \circ \sigma}\right) d \mu \\
& =-\int\left(\log \left(\frac{h}{h \circ \sigma} \frac{d \nu}{d \nu \circ \sigma}\right)\right) d \mu \\
& =-\int\left(f^{\prime}-P_{G}\left(f^{\prime}\right)+\log h-\log h \circ \sigma\right) d \mu \\
& =-\int f^{\prime} d \mu+P_{G}\left(f^{\prime}\right),
\end{aligned}
$$

ou seja $P_{G}\left(f^{\prime}\right)=P_{\mu}\left(f^{\prime}\right)$.

Seja $\bar{\mu} \in \mathcal{M}_{\sigma}(\Sigma)$ definida como $\bar{\mu}(E)=\mu\left(E \cap \Sigma^{\prime}\right)$ para todo $E \subset \Sigma$ mensurável. É fácil ver que $h(\bar{\mu})=h(\mu)$, portanto

$$
P_{G}\left(f^{\prime}\right)=P_{\mu}\left(f^{\prime}\right)=P_{\bar{\mu}}(f) \leq \sup \left\{P_{\eta}(f): \eta \in \mathcal{M}_{\sigma}(\Sigma)\right\}
$$

portanto $P_{G}(f) \leq \sup \left\{P_{\eta}(f): \eta \in \mathcal{M}_{\sigma}(\Sigma)\right\}+\epsilon$, dado que $\epsilon$ é arbitrário obtemos a desigualdade.

$(\geq)$ Podemos assumir s.p.d.g. $P_{G}(f)<\infty$, no caso $P_{G}(f)=\infty$ a desigualdade é trivial. Seja $\mu \in \mathcal{M}_{\sigma}(\Sigma)$ tal que $P_{\mu}(f)$ está bem definido. No caso $\int f d \mu=-\infty$ temos $P_{G}(f)>-\infty=P_{\mu}(f)$.

Suponhamos $\int f d \mu>-\infty$, seja $\alpha_{m}=\{[1],[2], \ldots,[m-1],[\geq m]\}$ onde $[\geq m]=\bigcup_{k \geq m}[k]$, portanto

$$
\lim _{m \rightarrow \infty} h\left(\mu, \alpha_{m}\right)=h(\mu) .
$$


Seja $A \subset \Sigma$ mensurável, definimos $S_{n} f(A):=\left.\sup \left(S_{n} f\right)\right|_{A}$, então

$$
\begin{aligned}
\frac{1}{n} H\left(\mu \mid \alpha_{m}^{n}\right)+\int f d \mu & =\frac{1}{n}\left(H\left(\mu \mid \alpha_{m}^{n}\right)+\int S_{n} f d \mu\right) \\
& =\frac{1}{n}\left(-\sum_{A \in \alpha_{m}^{n}} \mu(A) \log \mu(A)+\left.\sum_{A \in \alpha_{m}^{n}} \int\left(S_{n} f\right)\right|_{A} d \mu\right) \\
& \leq \frac{1}{n}\left(-\sum_{A \in \alpha_{m}^{n}} \mu(A) \log \mu(A)+\sum_{A \in \alpha_{m}^{n}} \mu(A) S_{n} f(A)\right) \\
& =\frac{1}{n} \sum_{A \in \alpha_{m}^{n}} \mu(A) \log \left(\frac{\exp \left(S_{n} f(A)\right)}{\mu(A)}\right) \\
& =\frac{1}{n} \sum_{B, C \in \alpha_{m}} \mu\left(B \cap \sigma^{-n} C\right) \sum_{A \subset B \cap \sigma^{-n} C} \mu\left(A \mid\left(B \cap \sigma_{m}^{-n} C\right)\right) \log \left(\frac{\exp \left(S_{n} f(A)\right)}{\mu(A)}\right) \\
& \leq \frac{1}{n} H\left(\mu \mid \alpha_{m} \vee \sigma^{-n} \alpha_{m}\right)+\frac{1}{n} \sum_{B, C \in \alpha_{m}} \mu\left(B \cap \sigma^{-n} C\right) \quad \sum_{A \subset B \cap \sigma^{-n} C} S_{n} f(A) \\
& \leq \frac{1}{n} H\left(\mu \mid \alpha_{m} \vee \sigma^{-n} \alpha_{m}\right)+\sum_{B, C \in \alpha_{m}} \mu\left(B \cap \sigma^{-n} C\right) P_{n}(B, C),
\end{aligned}
$$

onde

$$
P_{n}(B, C):=\frac{1}{n} \log \left(\sum_{\substack{A \subset B \cap \sigma^{-n} C \\ A \in \alpha_{m}^{n}}} \exp \left(S_{n} f(A)\right)\right) .
$$

Dado que $H(\mu \mid \mathcal{P} \vee \mathcal{Q}) \leq H(\mu \mid \mathcal{P})+H(\mu \mid \mathcal{Q})$, então

$$
\begin{aligned}
\frac{1}{n} H\left(\mu \mid \alpha_{m}^{n}\right)+\int f d \mu & \leq \frac{1}{n}\left(H\left(\mu \mid \alpha_{m}\right)+H\left(\mu \mid \sigma^{-n} \alpha_{m}\right)\right)+\sum_{B, C \in \alpha_{m}} \mu\left(B \cap \sigma^{-n} C\right) P_{n}(B, C) \\
& \leq O\left(\frac{2}{n} H\left(\mu \mid \alpha_{m}\right)\right)+\sum_{B, C \in \alpha_{m}} \mu\left(B \cap \sigma^{-n} C\right) P_{n}(B, C),
\end{aligned}
$$

portanto

$$
h\left(\mu, \alpha_{m}^{n}\right)+\int f d \mu \leq \limsup _{n \rightarrow \infty} \sum_{B, C \in \alpha_{m}} \mu\left(B \cap \sigma^{-n} C\right) P_{n}(B, C) .
$$

Se $B, C \neq[\geq m]$ ou seja $B=[b]$ e $C=[c]$ para $b, c<m$. Seja $\alpha=\{[a]: a \in \mathcal{A}\}$ a partição natural, portanto $\alpha$ é mais fina que $\alpha_{m}$, então substituindo em (3.5) segue

$$
P_{n}([b],[c])=\frac{1}{n} \log \left(\sum_{\substack{A \subset[b] \cap \sigma^{-n}[c] \\ A \in \alpha_{m}^{n}}} \exp \left(S_{n} f(A)\right)\right) \leq \frac{1}{n} \log \left(\sum_{\substack{[\omega] \subset[b] \cap \sigma^{-n}[c] \\[\omega] \in \alpha^{n}}} \exp \left(S_{n} f([\omega])\right)\right)
$$


dado que $\sigma$ é topologicamente transitivo e $V(f)<\infty$, existe $k \geq 1$ tal que

$$
\limsup _{n \rightarrow \infty} P_{n}([b],[c]) \leq \limsup _{n \rightarrow \infty} \frac{1}{n+k} \log \left(\sum_{\substack{\sigma^{n+k} x=x \\ x_{0}=b, x_{n}=c, x_{n+k}=b}} \exp \left(\sup \left(S_{n+k} f(x)\right)\right)\right) \leq P_{G}(f)
$$

Se $B=[\geq m]$ ou $C=[\geq m]$, então para cada $A \in \alpha_{m}^{n}$ tal que $A \subset B \cap \sigma^{-n} C$, existem $i, j, k \geq 0$ tais que $i+j+k=n \mathrm{e}$

$$
A=\bigcap_{l=0}^{i-1} \sigma^{-l}[\geq m] \cap \sigma^{-i}\left[\xi_{0}\right] \cap \ldots \cap \sigma^{-i-j+1}\left[\xi_{j-1}\right] \cap \bigcap_{l=i+j}^{n-1} \sigma^{-l}[\geq m],
$$

portanto

$$
S_{n} f(A) \leq(i+k) \sup f+S_{j} f\left(\sigma^{-i}\left[\xi_{0}\right] \cap \ldots \cap \sigma^{-i-j+1}\left[\xi_{j-1}\right]\right),
$$

além disso usando (3.5) concluímos que

$$
j P_{j}\left(\left[\xi_{0}\right],\left[\xi_{j-1}\right]\right) \leq S_{j} f\left(\left[\xi_{0}\right] \cap \ldots \cap \sigma^{-j+1}\left[\xi_{j-1}\right]\right),
$$

então substituindo em (3.5) obtemos o seguinte

$$
\begin{aligned}
P_{n}(B, C) & \leq \frac{1}{n} \log \left(\sum_{\substack{A \subset B \cap \sigma^{-n} C \\
A \in \alpha_{m}^{n}}} e^{(i+k) \sup f+S_{j} f\left(\sigma^{-i}\left[\xi_{0}\right] \cap \ldots \cap \sigma^{-i-j+1}\left[\xi_{j-1}\right]\right)}\right) \\
& \leq \frac{1}{n} \log \left(e^{S_{n} f\left(\cap_{l=0}^{i+k-1} \sigma^{-l}[\geq m]\right)}+\sum_{i=0}^{n-1} \sum_{j=1}^{n-i} \sum_{k=0}^{n-i-j} e^{(i+k) \sup f+S_{j} f\left(\sigma^{-i}\left[\xi_{0}\right] \cap \ldots \cap \sigma^{-i-j+1}\left[\xi_{j-1}\right]\right)}\right) \\
& =\sup f+\frac{1}{n} \log \left(\sum_{i=0}^{n-1} \sum_{j=1}^{n-i} \sum_{k=0}^{n-i-j} e^{S_{j} f\left(\sigma^{-i}\left[\xi_{0}\right] \cap \ldots \cap \sigma^{-i-j+1}\left[\xi_{j-1}\right]\right)}\right) \\
& =\sup f+\sum_{i=0}^{n-1} \sum_{j=1}^{n-i} \sum_{k=0}^{n-i-j} e^{j P_{j}\left(\sigma^{-i}\left[\xi_{0}\right], \sigma^{-i}\left[\xi_{j-1}\right]\right)}
\end{aligned}
$$


dado que $j P_{j}\left(\sigma^{-i}\left[\xi_{0}\right], \sigma^{-i}\left[\xi_{j-1}\right]\right) \leq P_{G}(f)<\infty$, então $P_{n}(B, C)<K_{0}<\infty$. Portanto

$$
\begin{aligned}
h\left(\mu, \alpha_{m}\right)+\int f d \mu & \leq \limsup _{n \rightarrow \infty}\left(\frac{1}{n} H\left(\mu \mid \alpha_{m}^{n}\right)+\int f d \mu\right) \\
& \leq \limsup _{n \rightarrow \infty}\left(\sum_{B, C \neq[\geq m]} P_{G}(f) \mu\left(B \cap \sigma^{-n} C\right)+\sum_{\begin{array}{c}
B=[\geq m], \\
\text { ou } C=[\geq m]
\end{array}} K_{0} \mu\left(B \cap \sigma^{-n} C\right)\right) \\
& \leq P_{G}(f)+K_{0}\left(\mu[\geq m]+\mu\left(\sigma^{-n}[\geq m]\right)\right) \\
& \leq P_{G}(f)+2 K_{0} \mu[\geq m] .
\end{aligned}
$$

Dado que a desigualdade é valida para todo $m \geq 0$ segue imediatamente que $P_{\mu}(f) \leq P_{G}(f)$.

Finalmente como $\mu$ é arbitraria, segue que $\sup \left\{P_{\mu}(f): \mu \in \mathcal{M}_{\sigma}(\Sigma)\right\} \leq P_{G}(f)$.

O lema 9 mostra que para nosso caso $P_{G}(f)=P_{t o p}(f)$, portanto de aqui para o frente sempre que falemos da pressão do potencial $f$ usaremos a notação $P_{G}(f)$, no entanto usaremos a definição de pressão que seja mais conveniente para cada caso.

Dizemos que uma medida $\mu \in \mathcal{M}_{\sigma}(\Sigma)$ é um estado de equilíbrio associado ao potencial $f$ se $\int f d \mu>-\infty$ e além disso

$$
P_{t o p}(f)=h(\mu)+\int f d \mu \text {. }
$$

Dizemos que uma medida $\mu \in \mathcal{M}_{\sigma}(\Sigma)$ é um estado de Gibbs invariante associado ao potencial $f$ se existe uma constante $C>1$ tal que para todo $x \in \Sigma$ e cada $n \geq 1$ é satisfeito

$$
\frac{\mu\left[x_{0} \ldots x_{n-1}\right]}{\exp \left(S_{n} f(x)-n P_{t o p}(f)\right)}=C^{ \pm 1}
$$

Quando $\Sigma$ é compacto os estados de equilíbrio e os estados de Gibbs invariantes coincidem. Além disso se $\Sigma$ tem matriz de incidência finitamente primitiva e $\bar{V}(f)<\infty$, Mauldin e Urbanski demonstraram em [14] que a constante $C$ pode ser escolhida como

$$
C=\exp (4 \bar{V}(f))
$$

No caso que o estado de equilíbrio seja único vamos denotar ele por $\mu_{f}$.

Lema 10. [22] Suponhamos que $P_{G}(f)<\infty$ e $V(f)<\infty$, então existe um potencial $f^{\prime}$ e uma constante $c$ tal que $f^{\prime} \backsim f+c^{2}$ e satisfaz $L_{f^{\prime}} 1=1, P_{G}\left(f^{\prime}\right)=0$.

\footnotetext{
${ }^{2} \mathrm{~A}$ constante $c$ é igual à pressão $-P_{G}(f)$ do potencial $f$.
} 


\subsection{Unicidade dos estados de equilíbrio}

Nesta seção vamos ver o teorema que garante a unicidade dos estados de equilíbrio quando o subshift $\Sigma$ é topologicamente transitivo com alfabeto enumerável e o potencial $f$ tem variação somável e pressão finita, este resultado vai nós ajudar a garantir a unicidade do estado de equilíbrio associado a cada um dos potenciais da família $(t f)_{t>1}$ que aparece no capitulo 5 , já que nessa construção somente podemos garantir a existência de estados de equilíbrio. O resultado foi demonstrado por Buzzi e Sarig.

Teorema 6 (Unicidade dos estados de equilíbrio). [6]

Seja $\Sigma$ um subshift de Markov topologicamente transitivo e $f: \Sigma \rightarrow \mathbb{R}$ um potencial limitado por acima, com $V(f)<\infty$ e $P_{G}(f)<\infty$. Então

1. $f$ tem no máximo um estado de equilíbrio $\mu_{f}$.

2. Se o estado de equilíbrio existe, então é igual à medida $R P F$ do $f$, e além disso tem entropia finita.

Demonstração. Seja $\mu$ um estado de equilíbrio associado ao potencial $f$, podemos supor s.p.d.g. $P_{G}(f)=0^{3}$. Pelo lema 10 existe $f^{\prime} \backsim f$ satisfazendo $L_{f^{\prime}} 1=1$ e $P_{G}\left(f^{\prime}\right)=0$, seja $\varphi$ tal que $f^{\prime}=f+\varphi-\varphi \circ \sigma$. Queremos demonstrar que $L_{f} e^{\varphi}=e^{\varphi}$ e $L_{f}^{*}\left(e^{-\varphi} \mu\right)=e^{-\varphi} \mu$, ou seja que $\mu$ é uma medida $R P F$ associada ao potencial $f$.

Afirmação 1. Temos $f, f^{\prime}, \varphi-\varphi \circ \sigma \in L^{1}(\mu)$, além disso $\int(\varphi-\varphi \circ \sigma) d \mu=0$.

Com efeito, dado que $0=h(\mu)+\int f d \mu$ segue que $\int f d \mu>-\infty$, e dado que sup $f<\infty$, então $f \in L^{1}(\mu)$, além disso como $f^{\prime} \leq 0$ e $f^{\prime}-f=\varphi-\varphi \circ \sigma$, então

$$
\int(\varphi-\varphi \circ \sigma) d \mu \in[-\infty, \infty)
$$

Seja $\mu=\int \mu_{P} d \hat{\mu}(P)$ a decomposição ergódica de $\mu$. Como $f \in L^{1}(\mu)$, então $f \in L^{1}\left(\mu_{P}\right)$ para $\hat{\mu}$-quase todo $P$, portanto $f-f^{\prime} \in L^{1}\left(\mu_{P}\right)$ ou $f-f^{\prime}=\infty$ em $\hat{\mu}$-quase todo $P$.

Então pelo teorema ergódico de Birkhoff temos que para $\mu_{P}$-quase todo $x \in \Sigma$

$$
\begin{aligned}
\int(\varphi-\varphi \circ \sigma) d \mu_{P} & =\lim _{n \rightarrow \infty} \frac{1}{n} S_{n}(\varphi-\varphi \circ \sigma)(x) \\
& =\lim _{n \rightarrow \infty} \frac{1}{n}\left(\varphi-\varphi \circ \sigma^{n}\right)(x) \\
& =0
\end{aligned}
$$

\footnotetext{
${ }^{3}$ Substituindo $f$ por $f-P_{G}(f)$ os estados de equilíbrio são os mesmos.
} 
ou seja $\int(\varphi-\varphi \circ \sigma) d \mu_{P}=0$ para $\hat{\mu}$-quase todo $P$, portanto $\int(\varphi-\varphi \circ \sigma) d \mu=0$, do anterior segue imediatamente que $f^{\prime} \in L^{1}(\mu)$.

Afirmação 2. Existe $I \subset \mathbb{N}$ tal que $\mu=\sum_{i \in I} p_{i} \mu_{i}, \sum_{i \in I} p_{i}=1$, e cada uma das medidas $\mu_{i}$ é um estado de equilíbrio ergódico. Além disso existe $a_{i} \in \mathcal{A}$ tal que $\left[a_{i}\right]$ é um conjunto sweep-out para $\mu_{i}$, ou seja para $\mu_{i}$-quase todo ponto $x \in \Sigma$ existem infinitos valores de $n$ tais que $\sigma^{n} x \in\left[a_{i}\right]$.

Com efeito, seja $\mu=\int \mu_{P} d \hat{\mu}(P)$ a decomposição ergódica de $\mu$ e seja $\left\{a_{0}, a_{1}, \ldots\right\} \subset \mathcal{A}$ tal que $\mu\left[a_{i}\right]>0$ para todo $i \in \mathbb{N}$. Definimos o conjunto

$$
\mathcal{Q}_{i}=\left\{P \in \mathcal{P}: \mu_{P}\left[a_{0}\right]=\ldots=\mu_{P}\left[a_{i-1}\right]=0, \mu_{P}\left[a_{i}\right]>0\right\},
$$

portanto segue da ergodicidade das $\mu_{P}$ que $\mathcal{Q}_{i} \cap \mathcal{Q}_{j}=\emptyset$ para cada $i \neq j$, além disso se $p_{i}=\hat{\mu}\left(\mathcal{Q}_{i}\right)$ para cada $i \in \mathbb{N}$, então é satisfeito $\sum_{i \in \mathbb{N}} p_{i}=1$. Seja $I=\left\{i \in \mathbb{N}: p_{i} \neq 0\right\}$, para cada $i \in I$ definimos a medida $\mu_{i}$ como $\mu_{i}(E)=\mu\left(E \mid \pi^{-1} \mathcal{Q}_{i}\right)$ onde $\pi: \Sigma \rightarrow \mathcal{P}$ é a projeção natural, portanto $\mu=\sum_{i \in I} p_{i} \mu_{i}$, além disso pela construção das $\mu_{i}$ cada um dos cilindros $\left[a_{i}\right]$ é um conjunto sweep-out para $\mu_{i}$.

Suponhamos que $|I|<\infty$, como $\mu$ é um estado de equilíbrio e $P_{G}(f)=0$, então $h(\mu)<\infty$. Além disso dado que a função entropia é afim, ou seja $h(\mu)=\sum_{i \in I} p_{i} h\left(\mu_{i}\right)$, então $h\left(\mu_{i}\right)<\infty$ para todo $i \in I$, portanto $h\left(\mu_{i}\right)+\int f d \mu_{i}$ esta bem definido e além disso

$$
\sum_{i \in I} p_{i}\left(h\left(\mu_{i}\right)+\int f d \mu_{i}\right)=h(\mu)+\int f d \mu=0,
$$

ou seja cada uma das medidas $\mu_{i}$ é um estado de equilíbrio.

Suponhamos $|I|=\infty$, para cada $N \in \mathbb{N}$ definimos $q_{N}=\sum_{i>N} p_{i}$, dado que $q_{N} \neq 0$ podemos definir $\mu_{N+1}^{*}=\frac{1}{q_{N}} \sum_{i>N} p_{i} \mu_{i}$, então $\mu=q_{N} \mu_{N+1}^{*}+\sum_{i \leq N} p_{i} \mu_{i}$, do qual segue

$$
h(\mu)=q_{N} h\left(\mu_{N+1}^{*}\right)+\sum_{i \leq N} p_{i} h\left(\mu_{i}\right)
$$

ou seja $h\left(\mu_{N+1}^{*}\right)<\infty$, e $h\left(\mu_{i}\right)<\infty$ para cada $i \leq N$, portanto

$$
q_{N} h\left(\mu_{N+1}^{*}\right)+\mu_{N+1}^{*}(f)+\sum_{i \leq N} p_{i}\left(h\left(\mu_{i}\right)+\int f d \mu_{i}\right)=h(\mu)+\int f d \mu=0,
$$

ou seja $\mu_{N+1}^{*}$ e cada uma das medidas $\mu_{i} \operatorname{com} i \leq N$ são estados de equilíbrio. Finalmente como $N$ é arbitrário podemos concluir que $\mu_{i}$ é estado de equilíbrio para todo $i \in I$. 
Afirmação 3. Para cada $i \in I$ temos $h\left(\mu_{i}\right)=-\int\left(\log \frac{d \mu_{i}}{d \mu_{i} \circ \sigma}\right) d \mu_{i}$.

A afirmação 3 não é consequência imediata da formula de Rokhlin dado que $\Sigma$ não é compacto, portanto $H\left(\mu_{i} \mid \alpha^{n}\right)$ pode ser infinita para algum $n$. Fixemos $i$ e sejam $a_{i}$ e $\mu_{i}$ como foram construídos na afirmação 2, vamos definir $\bar{\sigma}$ como $\bar{\sigma}(x)=\sigma^{\varphi_{a_{i}}(x)}(x)$. Para $E \subset \Sigma$ mensurável definimos $\bar{\mu}_{i}(E)=$ $\mu_{i}\left(E \mid\left[a_{i}\right]\right)$, dado que a medida $\mu_{i}$ é $\sigma$-invariante e que $\bar{\sigma}^{-1}\left[a_{i}\right]=\sigma^{-\varphi_{a_{i}}}\left[a_{i}\right]=\left[a_{i}\right]$, então a medida $\bar{\mu}_{i}$ é $\bar{\sigma}$-invariante.

Seja $\beta=\left\{\left[a_{i} \xi_{1} \ldots \xi_{n-1} a_{i}\right]: n \geq 1, \xi_{k} \neq a_{i}\right\} \backslash\{\emptyset\}$ uma partição de $\Sigma$, vamos mostrar que $H\left(\bar{\mu}_{i} \mid \beta\right)<\infty$. Para isso definimos a medida de Bernoulli $\bar{\mu}_{B}^{i}$ sobre $\left[a_{i}\right]$ como

$$
\bar{\mu}_{B}^{i}\left(\bigcap_{j=0}^{n-1} \bar{\sigma}^{-j}\left[\omega_{j}\right]\right)=\prod_{j=0}^{n-1} \bar{\mu}_{i}\left[\omega_{j}\right],
$$

onde $\left[\omega_{j}\right] \in \beta$ para cada $j$. Dado que $\bar{\mu}_{B}^{i}[\omega]=\bar{\mu}_{i}[\omega]$ para todo $[\omega] \in \beta$ e a medida $\bar{\mu}_{B}^{i}$ é uma medida de Bernoulli, então

$$
h\left(\bar{\mu}_{B}^{i}\right)=H\left(\bar{\mu}_{B}^{i} \mid \beta\right)=H\left(\bar{\mu}_{i} \mid \beta\right),
$$

portanto é suficiente demonstrar que $h\left(\bar{\mu}_{B}^{i}\right)<\infty$. Definimos a medida $\sigma$-invariante $\mu_{B}^{i}$ como

$$
\mu_{B}^{i}(E)=\mu_{i}\left[a_{i}\right] \int 1_{\left[a_{i}\right]}\left(\sum_{j=0}^{\varphi_{a_{i}}-1} 1_{E} \circ \sigma^{j}\right) d \bar{\mu}_{B}^{i}
$$

para cada $E \subset \Sigma$ mensurável. Usando a formula de Kâc e (3.10) temos o seguinte

$$
\begin{aligned}
\mu_{B}^{i}(\Sigma) & =\mu_{i}\left[a_{i}\right] \int 1_{\left[a_{i}\right]} \varphi_{a_{i}} d \bar{\mu}_{B}^{i} \\
& =\mu_{i}\left[a_{i}\right] \sum_{[\omega] \in \beta}|\omega| \bar{\mu}_{B}^{i}[\omega] \\
& =\mu_{i}\left[a_{i}\right] \int \varphi_{a_{i}} d \bar{\mu}_{i} \\
& =\mu_{i}(\Sigma)=1 .
\end{aligned}
$$

Dado que $\bar{\mu}_{B}^{i}$ é Bernoulli (3.8), então também é $\bar{\sigma}$-ergódica, além disso se uma função $g$ é $\sigma$ invariante, então a função $\left.g\right|_{\left[a_{i}\right]}$ é $\bar{\sigma}$-invariante, e pela ergodicidade da medida $\bar{\mu}_{B}^{i}$ segue que $\left.g\right|_{\left[a_{i}\right]}$ é constante $\bar{\mu}_{B}^{i}$-quase sempre, então segue de (3.8) que $\left.g\right|_{\left[a_{i}\right]}$ é constante $\bar{\mu}_{i}$-quase sempre, além disso dado que $\left[a_{i}\right]$ é um conjunto sweep-out para $\mu_{i}$, segue da formula de Kâc que $g$ é constante $\mu_{i^{-}}$ quase sempre, e como todo conjunto de $\mu_{i}$-medida total tem $\mu_{B}^{i}$-medida total, então $g$ é constante $\mu_{B}^{i}$-quase sempre e portanto $\mu_{B}^{i}$ é $\sigma$-ergódica. 
Seja $M=\sup _{n \geq 1} V_{n+1}\left(S_{n} f\right)$ e $\bar{f}=\sum_{j=0}^{\varphi_{a_{i}}-1} f \circ \sigma^{j}$, para cada $[\omega] \in \beta$ fixemos $x_{[\omega]} \in[\omega]$, então para todo $y \in[\omega]$

$$
|| \bar{f}(y)|-| \bar{f}\left(x_{[\omega]}\right)|| \leq\left|\bar{f}(y)-\bar{f}\left(x_{[\omega]}\right)\right| \leq V_{|\omega|}\left(S_{|\omega|} f\right) \leq M<\infty .
$$

Portanto o valor médio de $|\bar{f}|$ sobre o cilindro $[\omega]$ está no intervalo $\left[\left|\bar{f}\left(x_{[\omega]}\right)\right|-M,\left|\bar{f}\left(x_{[\omega]}\right)\right|+M\right]$, então para todo $[\omega] \in \beta$

$$
\left|\frac{1}{\bar{\mu}_{i}[\omega]} \int 1_{[\omega]}\right| \bar{f}\left|d \bar{\mu}_{i}-\frac{1}{\bar{\mu}_{B}^{i}[\omega]} \int 1_{[\omega]}\right| \bar{f}\left|d \bar{\mu}_{B}^{i}\right| \leq 2 M
$$

e dado que $\bar{\mu}_{i}[\omega]=\bar{\mu}_{B}^{i}[\omega]$ para todo $[\omega] \in \beta$, temos $\bar{f} \in L^{1}\left(\bar{\mu}_{i}\right)$ se, e somente se $\bar{f} \in L^{1}\left(\bar{\mu}_{B}^{i}\right)$. Agora $\bar{f} \in L^{1}\left(\bar{\mu}_{i}\right)$ porque pela formula de Kâc temos

$$
\int|\bar{f}| d \bar{\mu}_{i} \leq \int \overline{|f|} d \bar{\mu}_{i}=\frac{1}{\mu_{i}\left[a_{i}\right]} \int|f| d \mu_{i}<\frac{1}{p_{i} \mu_{i}\left[a_{i}\right]} \int|f| d \mu<\infty
$$

portanto $\bar{f} \in L^{1}\left(\bar{\mu}_{B}^{i}\right)$, do qual segue $f \in L^{1}\left(\mu_{B}^{i}\right)$, então $h\left(\mu_{B}^{i}\right)+\int f d \mu_{B}^{i}$ está bem definido e pelo principio variacional $h\left(\mu_{B}^{i}\right)+\int f d \mu_{B}^{i} \leq P_{G}(f)=0$, ou seja $h\left(\mu_{B}^{i}\right) \leq-\int f d \mu_{B}^{i}<\infty$, além disso a formula de Abramov diz o seguinte $h\left(\bar{\mu}_{B}^{i}\right)=\frac{1}{\mu_{B}^{i}\left[a_{i}\right]} h\left(\mu_{B}^{i}\right)<\infty$, então segue de (3.9) que $H\left(\bar{\mu}_{i} \mid \beta\right)<\infty$.

Finalmente pela formula de Rokhlin e usando novamente a formula de Kâc, segue que

$$
\begin{aligned}
\frac{1}{\mu_{i}\left[a_{i}\right]} h\left(\mu_{i}\right) & =h\left(\bar{\mu}_{i}\right) \\
& =-\int \log \frac{d \bar{\mu}_{i}}{d \bar{\mu}_{i} \circ \sigma} d \bar{\mu}_{i} \\
& =\frac{-1}{\mu_{i}\left[a_{i}\right]} \int 1_{\left[a_{i}\right]} \log \frac{d \mu_{i}}{d \mu_{i} \circ \sigma^{\varphi a_{i}}} d \mu_{i} \\
& =\frac{-1}{\mu_{i}\left[a_{i}\right]} \int 1_{\left[a_{i}\right]}\left(\sum_{j=0}^{\varphi_{a_{i}}-1} \log \frac{d \mu_{i}}{d \mu_{i} \circ \sigma} \circ \sigma^{j}\right) d \mu_{i} \\
& =\frac{-1}{\mu_{i}\left[a_{i}\right]} \int \log \frac{d \mu_{i}}{d \mu_{i} \circ \sigma} d \mu_{i},
\end{aligned}
$$

e com isso a afirmação 3 é demonstrada.

Afirmação 4. Para cada $i \in I$ temos $\log \frac{d \mu_{i}}{d \mu_{i} \circ \sigma}=f^{\prime}$.

Para cada $i \in I$ definimos $f_{i}^{\prime}=\log \frac{d \mu_{i}}{d \mu_{i} \circ \sigma}$, portanto $L_{f_{i}^{\prime}} 1=1$, além disso segue do afirmação 3 e 
das propriedades da função $\log ^{4}$ que

$$
\begin{aligned}
0=h\left(\mu_{i}\right)+\int f d \mu_{i} & =h\left(\mu_{i}\right)+\int f^{\prime} d \mu_{i} \\
& =\int f^{\prime}-f_{i}^{\prime} d \mu_{i} \\
& =\int f^{\prime}-f_{i}^{\prime} d\left(L_{f_{i}^{\prime}}^{*} \mu_{i}\right) \\
& =\int L_{f_{i}^{\prime}}\left(f^{\prime}-f_{i}^{\prime}\right) d \mu_{i} \\
& \leq \int \log \left(L_{f^{\prime}} 1\right) d \mu_{i}=0,
\end{aligned}
$$

portanto $L_{f_{i}^{\prime}}\left(f^{\prime}-f_{i}^{\prime}\right)(x)=\log \left(L_{f^{\prime}} 1\right)=0$ para $\mu_{i}$-quase todo ponto $x \in \Sigma$, ou seja

$$
\sum_{\sigma y=x} \exp \left(f_{i}^{\prime}(y)\right)\left(f^{\prime}-f_{i}^{\prime}\right)(y)=\log \left(\sum_{\sigma y=x} \exp \left(f^{\prime}(y)-f_{i}^{\prime}(y)\right) \exp \left(f_{i}^{\prime}(y)\right)\right)
$$

do qual segue $\exp \left(\left(f^{\prime}-f_{i}^{\prime}\right)(y)\right)=c(x)^{5}$. Dado que $L_{f_{i}^{\prime}}\left(f^{\prime}-f_{i}^{\prime}\right)(x)=0$, então $\log (c(x))=0$ para $\mu_{i}$-quase todo $x$. Em conclusão se $y \in \sigma^{-1}\{x\}$ temos $f^{\prime}(y)=f_{i}^{\prime}(y)$ para $\mu_{i}$-quase todo $x$ e com isso é demonstrada a afirmação 4 .

Para cada $i \in I$ temos $\log \frac{d \mu_{i}}{d \mu_{i} \circ \sigma}=f^{\prime}$, portanto $L_{f^{\prime}}^{*} \mu_{i}=\mu_{i}$, e dado que $f^{\prime}-\varphi=f-\varphi \circ \sigma$ segue que

$$
\begin{aligned}
\int g(x) d\left(L_{f}^{*}\left(e^{-\varphi} \mu_{i}\right)\right)(x) & =\int e^{-\varphi(x)}\left(L_{f} g\right)(x) d \mu_{i}(x) \\
& =\int \sum_{\sigma y=x} e^{f(y)-\varphi(\sigma y)} g(y) d \mu_{i}(x) \\
& =\int L_{f^{\prime}}\left(e^{-\varphi} g\right)(x) d \mu_{i}(x) \\
& =\int e^{-\varphi(x)} g(x) d \mu_{i}(x) .
\end{aligned}
$$

Como $\mu=\sum_{i \in I} p_{i} \mu_{i}$ e $L_{f}^{*}\left(e^{-\varphi} \mu_{i}\right)=e^{-\varphi} \mu_{i}$ para cada $i \in I$, então pela linearidade do operador $L_{f}^{*}$ temos $L_{f}^{*}\left(e^{-\varphi} \mu\right)=e^{-\varphi} \mu$, e dado que $L_{f^{\prime}} 1=1$ segue que $L_{f} e^{\varphi}=e^{\varphi}$, ou seja $\mu=e^{\varphi}\left(e^{-\varphi} \mu\right)$ é uma medida $R P F$.

Suponhamos que o $f$ tem mais do que um estado de equilíbrio, como todo estado de equilíbrio pode ser escrito como combinação linear de estados de equilíbrio ergódicos (afirmação 2), então existem ao menos dois estados de equilíbrio ergódicos $\mu_{1}$ e $\mu_{2}$ os quais satisfazem $L_{f}^{*}\left(e^{-\varphi} \mu_{1}\right)=e^{-\varphi} \mu_{1}$ e $L_{f}^{*}\left(e^{-\varphi} \mu_{2}\right)=e^{-\varphi} \mu_{2}$. Seja $\mu=\frac{1}{2}\left(\mu_{1}+\mu_{2}\right)$, então a medida $\mu$ não é ergódica e portanto $e^{-\varphi} \mu$ não

\footnotetext{
${ }^{4}$ Se $\sum_{y} p_{y}=1$, então $\sum_{y} p_{y} \log z_{y} \leq \log \left(\sum_{y} p_{y} z_{y}\right)$.

${ }^{5}$ Se $\sum_{y} p_{y}=1$ e $\sum_{y} p_{y} \log z_{y}=\log \left(\sum p_{y} z_{y}\right)$, então $z_{y}=c$ para todo $y$.
} 
é ergódica, porém $L_{f}^{*}\left(e^{-\varphi} \mu\right)=e^{-\varphi} \mu$ contradizendo o lema 8 .

Teorema 7. [19] Sejam $f^{\prime}$ e $f$ dois potenciais com variação somável tais que $P_{G}\left(f^{\prime}\right), P_{G}(f)<\infty$ $e \sup f^{\prime}, \sup f<\infty$. Então $f^{\prime} \backsim f+c$ se, e somente se $\mu_{f^{\prime}}=\mu_{f}$.

Demonstração. $(\Rightarrow)$ Suponhamos que $f^{\prime} \backsim f+c$ via a função $\varphi$. Dado que sup $f<\infty$ e $\mu_{f}$ é ergódica, pelo teorema ergódico de Birkhoff temos para $\mu_{f}$-quase todo ponto $x \in \Sigma$

$$
\int f d \mu_{f}=\lim _{n \rightarrow \infty} \frac{1}{n} S_{n} f(x)
$$

e dado que $\sup f^{\prime}<\infty$ para $\mu_{f}$-quase todo ponto $x \in \Sigma$

$$
\int f^{\prime} d \mu_{f}=\lim _{n \rightarrow \infty} \frac{1}{n} S_{n} f^{\prime}(x)
$$

Portanto

$$
\int f^{\prime} d \mu_{f}=\lim _{n \rightarrow \infty} \frac{1}{n} S_{n} f^{\prime}(x)=\lim _{n \rightarrow \infty} \frac{1}{n}\left(S_{n} f(x)+\varphi(x)-\varphi\left(\sigma^{n} x\right)+n c\right)=\int f d \mu_{f}+c .
$$

Dado que $\mu_{f}$ é um estado de equilíbrio associado ao potencial $f$ temos

$$
h\left(\mu_{f}\right)+\int f^{\prime} d \mu_{f}=h\left(\mu_{f}\right)+\int f d \mu_{f}+c=P_{G}(f)+c=P_{G}\left(f^{\prime}\right),
$$

ou seja $\mu_{f}$ também é um estado de equilíbrio associado ao potencial $f^{\prime}$, então segue da unicidade dos estados de equilíbrio que $\mu_{f^{\prime}}=\mu_{f}$.

$(\Leftarrow)$ Suponhamos que $\mu_{f^{\prime}}=\mu_{f}=\mu$, s.p.d.g. vamos supor $P_{G}(f)=P_{G}\left(f^{\prime}\right)=0$. Como $\mu$ é um estado de equilíbrio associado ao potencial $f$, então $\mu=h d \nu$ onde $L_{f} h=h$ e $L_{f}^{*} \nu=\nu$, além disso pelo teorema generalizado de Ruelle-Perron-Frobenious (ver [19]) para cada $a \in \mathcal{A}$ existe $C_{a}>0$ tal que $\left.h\right|_{[a]}=C_{a}^{ \pm 1}$. Definimos $M:=\exp \left(\sup _{n \geq 1} V_{n+1}\left(S_{n} f\right)\right)$, então para todo $x \in \Sigma$ e $\omega=x_{0} \ldots x_{n-1}$ temos

$$
\begin{aligned}
\mu[\omega] & =C_{x_{0}}^{ \pm 1} \nu[\omega] \\
& =C_{x_{0}}^{ \pm 1} \int\left(L_{f}^{n} 1_{[\omega]}\right)(y) d \nu(y) \\
& =C_{x_{0}}^{ \pm 1} \int \exp \left(S_{n} f(\omega y)\right) 1_{\sigma\left[x_{n-1}\right]}(y) d \nu(y) \\
& =\left(M C_{x_{0}}\right)^{ \pm 1} \nu\left(\sigma\left[x_{n-1}\right]\right) \exp \left(S_{n} f(x)\right) .
\end{aligned}
$$


Seja $y \in \Sigma$ tal que $\sigma^{m} y=y$, então

$$
\begin{aligned}
\frac{1}{m} S_{m} f(y)=\lim _{n \rightarrow \infty} \frac{1}{m n} S_{m n} f(y) & =\lim _{n \rightarrow \infty} \frac{1}{n}\left(\log \left(\mu\left[y_{0} \ldots y_{m n-1}\right]\right)-\log \left(\nu\left(\sigma\left[x_{m n-1}\right]\right)\right) \pm \log \left(M C_{x_{0}}\right)\right) \\
& =\lim _{n \rightarrow \infty} \frac{1}{n}\left(\log \left(\mu\left[y_{0} \ldots y_{m n-1}\right]\right)\right)
\end{aligned}
$$

As mesmas contas que acima, mas agora usando que $\mu$ é um estado de equilíbrio associado ao potencial $f^{\prime}$ e portanto $\mu=h^{\prime} d \nu^{\prime}$ onde $L_{f^{\prime}} h^{\prime}=h^{\prime}$ e $L_{f^{\prime}}^{*} \nu^{\prime}=\nu^{\prime}$ mostram que

$$
\frac{1}{m} S_{m} f^{\prime}(y)=\lim _{n \rightarrow \infty} \frac{1}{n}\left(\log \left(\mu\left[y_{0} \ldots y_{m n-1}\right]\right)\right)
$$

ou seja $S_{m} f^{\prime}(x)=S_{m} f(x)$, portanto segue do teorema 5 que $f^{\prime} \backsim f$. 


\section{Capítulo 4}

\section{Limite em temperatura zero sobre}

\section{compactos e otimização ergódica}

Seja $\Sigma$ um subshift de Markov e $f$ um potencial fixado, a otimização ergódica estuda existência e propriedades das medidas invariantes $\mu$ que maximizam $\int f d \mu$. Observemos que quando $\Sigma$ é compacto a existência destas medidas é imediata, porém quando $\Sigma$ não é compacto, a priori, não podemos garantir existência de medidas $f$-maximizantes.

Na primeira parte deste capítulo vamos ver um resultado demonstrado por Bissacot e Freire que garante existência de medidas maximizantes quando o subshift é não-compacto ${ }^{1}$ e topologicamente transitivo. Além disso este resultado garante que o suporte de todas as medidas maximizantes está contido num subshift compacto.

Na segunda parte do capítulo vamos estudar uma caracterização do limite em temperatura zero no caso compacto demonstrado por Leplaideur, além disso vamos mostrar que a medida limite é uma medida maximizante. Este resultado vai ser muito importante no capitulo 5 pois usando ele é que podemos caracterizar o limite em temperatura zero no caso topologicamente transitivo com alfabeto enumerável.

\subsection{Otimização ergódica}

No caso compacto sempre existem medidas maximizantes como consequência da compacidade do espaço $\mathcal{M}_{\sigma}(\Sigma)$, porém no caso não-compacto a existência destas medidas não é imediata, o seguinte resultado demonstrado por Bissacot e Freire é o primeiro que garante existência destas medidas num contexto além do que o caso de subshifts de Markov finitamente primitivos.

\footnotetext{
${ }^{1}$ Vamos estudar somente o caso onde o subshift tem alfabeto enumerável.
} 
Seja

$$
\beta=\sup \left\{\int f d \mu: \mu \in \mathcal{M}_{\sigma}(\Sigma)\right\}
$$

dizemos que uma medida $\mu \in \mathcal{M}_{\sigma}(\Sigma)$ é $f$-maximizante se $\int f d \mu=\beta$. Vamos denotar por $\mathcal{M}_{\text {max }}(f)$ o conjunto das medidas $f$-maximizantes.

Teorema 8. [3] Seja $\Sigma$ um subshift de Markov topologicamente transitivo e $f: \Sigma \rightarrow \mathbb{R}$ um potencial coercivo com $\bar{V}(f)<\infty$. Então existe um conjunto finito $F \subset \mathcal{A}$ tal que

$$
\beta=\sup \left\{\int f d \mu: \mu \in \mathcal{M}_{\sigma}\left(\Sigma_{F}\right)\right\}<\infty
$$

onde $\Sigma_{F} \subset \Sigma$ é o menor subshift topologicamente transitivo, compacto, invariante, que contém o alfabeto $F$. Portanto $\mathcal{M}_{\max }(f) \neq \emptyset$ e além disso para cada $\mu \in \mathcal{M}_{\max }(f)$ temos $\operatorname{supp}(\mu) \subset \Sigma_{F}$.

Demonstração. Seja $\mathcal{M}_{\text {per }}(\Sigma)$ o conjunto das medidas periódicas $\sigma$-invariantes de $\Sigma^{2}$. É sabido que $\overline{\mathcal{M}_{\text {per }}(\Sigma)}=\mathcal{M}_{\text {erg }}(\Sigma)$, portanto usando o teorema de decomposição ergódica concluímos que

$$
\beta=\sup \left\{\int f d \eta: \eta \in \mathcal{M}_{p e r}(\Sigma)\right\}
$$

Vamos denotar a media ergódica do $f$ como $\beta(x)=\lim _{n \rightarrow \infty} \frac{1}{n} S_{n} f(x)$, observe que no caso $x \in \operatorname{Per}_{p}(\Sigma)$ o limite sempre existe, além disso $\beta(x)=\frac{1}{p} S_{p} f(x)$, portanto (4.2) pode ser reescrito da forma

$$
\beta=\sup \{\beta(x): x \in \operatorname{Per}(\Sigma)\}
$$

A ideia da prova do teorema é construir um conjunto finito $F \subset \mathcal{A}$ tal que

$$
\beta=\sup \left\{\beta(x): x \in \operatorname{Per}\left(\Sigma_{F}\right)\right\}
$$

Dado qualquer $x \in \Sigma$ dizemos que $x$ começa no valor $a$ se, e somente se $x_{j} \geq a$ para todo $j \in \mathbb{N}$.

Afirmação 1. Dado $\epsilon>0$ existe $I_{1} \in \mathbb{N}$ tal que se $x$ começa em $a \geq I_{1}$, então $\frac{1}{n} S_{n} f(x)<\beta-\epsilon$ para todo $n \in \mathbb{N}$. Em particular se $x \in \operatorname{Per}(\Sigma)$, então $\beta(x)<\beta-\epsilon$.

Seja $F_{1}^{\prime}=\left\{1, \ldots, I_{1}-1\right\}$, para cada dupla $a, b \in F_{1}^{\prime}$ escolhemos uma palavra $\omega_{a b}$ tal que $\left[a \omega_{a b} b\right] \neq \emptyset$, definimos $P_{0}=\max \left\{\left|\omega_{a b}\right|: a, b \in F_{1}^{\prime}\right\}$, seja $C_{1}^{\prime}$ o conjunto de símbolos que aparecem ao menos uma vez em alguma das palavras $\omega_{a b}$ com $a, b \in F_{1}^{\prime}$, portanto $\left|C_{1}^{\prime}\right|<\infty$, e seja $F_{1}=F_{1}^{\prime} \cup C_{1}^{\prime}$, observemos que $\left|F_{1}\right|<\infty$ e além disso $\Sigma_{F_{1}}$ é topologicamente transitivo.

\footnotetext{
${ }^{2}$ Medidas da forma $\eta=\frac{1}{p} \sum_{j=0}^{p-1} \delta_{\sigma^{j} x}$ onde $x \in \operatorname{Per}_{p}(\Sigma)$.
} 
Vamos fazer uma nova restrição no alfabeto $\mathcal{A}$. Sejam $\bar{V}(f)=\sum_{n \geq 1} V_{n}(f)<\infty$,

$$
\begin{aligned}
& C_{1}:=-\left(P_{0}|\min f|_{\Sigma_{F_{1}}}\left|+\left(P_{0}-1\right)\right| \beta \mid+2 \bar{V}(f)\right), \\
& C_{2}:=\beta-\epsilon-\bar{V}(f),
\end{aligned}
$$

definimos $I_{2} \geq I_{1}$ como o menor natural que satisfaz

$$
\left.\sup f\right|_{[a]}<C:=\min \left\{C_{1}, C_{2}\right\}, \forall a \geq I_{2} .
$$

Seja $F_{2}^{\prime}=\left\{1, \ldots, I_{2}-1\right\}$, para cada par $a, b \in F_{2}^{\prime}$ escolhemos uma palavra $\omega_{a b}$ tal que $\left[a \omega_{a b} b\right] \neq \emptyset$, seja $C_{2}^{\prime}$ o conjunto de símbolos que aparecem ao menos uma vez em alguma das palavras $\omega_{a b}$ com $a, b \in F_{2}^{\prime}$ e $F_{2}=F_{2}^{\prime} \cup C_{2}^{\prime}$, observemos que $F_{1} \subset F_{2},\left|F_{2}\right|<\infty$, e além disso $\Sigma_{F_{2}}$ é topologicamente transitivo.

Para $x \in \Sigma$ e $\omega=x_{l} \ldots x_{l+m}$, definimos

$$
\begin{gathered}
\kappa(l, m \mid x)=\kappa(\omega \mid x):=\frac{1}{m+1} S_{m+1} f\left(\sigma^{l} x\right), \\
\kappa_{r}(l, m \mid x)=\kappa_{r}(\omega \mid x):=\frac{1}{r+2}\left(f\left(\sigma^{l+m} x\right)+S_{r+1} f\left(\sigma^{l} x\right)\right), \text { se } r<m .
\end{gathered}
$$

Afirmação 2. Seja $x \in \operatorname{Per}_{p}(\Sigma)$ para algum $p \geq 1$, tal que $\beta(x) \geq \beta-\epsilon$ e $x \notin \Sigma_{F_{2}}$. Então existe ao menos uma palavra $x_{l} \ldots x_{l+m}$ em $x$ tal que:

i) $\kappa(l, m \mid x) \geq \beta(x)$;

ii) $x_{l} \leq I_{1}$, e $x_{l+m} \geq I_{2}$;

iii) $x_{l+j}<I_{2}$ para cada $j \in\{0, \ldots, m-1\}$.

iv) Se $r<m$ é o maior inteiro tal que $x_{l+r}<I_{1}$, então $\kappa(l, m \mid x) \leq \kappa_{r}(l, m \mid x)$.

Afirmação 3. Seja $x \in \operatorname{Per}(\Sigma)$ tal que $x \notin \Sigma_{F_{2}}$ e $\beta(x) \geq \beta-\epsilon$, então existe $z \in \operatorname{Per}\left(\Sigma_{F_{2}}\right)$ tal que $\beta(z)>\beta(x)$.

Seja $\left(x^{n}\right)_{n \in \mathbb{N}} \subset \operatorname{Per}(\Sigma)$ tal que $\lim _{n \rightarrow \infty} \beta\left(x^{n}\right)=\beta$, então podemos assumir que $\beta\left(x^{n}\right) \geq \beta-\epsilon$. Escolhendo $F=F_{2}$, pela afirmação 3 temos existe $\left(z^{n}\right)_{n \in \mathbb{N}} \subset \operatorname{Per}\left(\Sigma_{F}\right)$ tal que $\beta\left(z^{n}\right) \geq \beta\left(x^{n}\right)$ para todo $n \in \mathbb{N}$, portanto $\lim _{n \rightarrow \infty} \beta\left(z^{n}\right)=\beta$. O anterior implica

$$
\beta=\sup \left\{\beta(x): x \in \operatorname{Per}\left(\Sigma_{F}\right)\right\},
$$


e dado que $\Sigma_{F}$ é compacto podemos garantir que existe pelo menos uma medida maximizante, além disso o suporte dessa medida está contido em $\Sigma_{F}$.

Agora vamos demonstrar que toda $\mu \in \mathcal{M}_{\max }(f)$ satisfaz $\operatorname{supp}(\mu) \subset \Sigma_{F}$. Sabemos pela afirmação 3 que $\mathcal{M}_{\max }(f) \neq \emptyset$ e que toda medida periódica maximal tem seu suporte contido em $\Sigma_{F}$, portanto é suficiente demonstrar que toda $\mu \in \mathcal{M}_{\max }(f)$ não periódica satisfaz $\operatorname{supp}(\mu) \subset \Sigma_{F}$.

Suponhamos que existe $\mu \in \mathcal{M}_{\max }(f)$ não periódica tal que $\operatorname{supp}(\mu) \not \subset \Sigma_{F}$, podemos supor s.p.d.g. $\mu \in \mathcal{M}_{\text {erg }}(\Sigma)^{3}$. A ideia é construir uma medida periódica $\sigma$-invariante tal que a integral com respeito a essa medida é estritamente maior que $\beta$ o qual é absurdo. Vamos supor $\beta=0$, como $\Sigma$ é topologicamente transitivo, então o conjunto $\Omega$ de pontos recorrentes de $\Sigma$ é residual, portanto podemos escolher $x \in \operatorname{supp}(\mu) \cap \Sigma_{F}^{c}$ um ponto genérico recorrente tal que $\beta(x)=0$.

Dado que $x \in \operatorname{supp}(\mu) \cap \Sigma_{F}^{c}$, existe um simbolo $I \geq I_{2}$ na expressão de $x$. Queremos modificar $x$ num novo ponto $z \in \operatorname{Per}_{p}(\Sigma)$ tal que $\beta(z)>0$, e dado que $\beta(z)=\int f d \nu$ para $\nu=\frac{1}{p} \sum_{j=0}^{p-1} \delta_{\sigma^{j} z}$ isso contradiz o fato que $\mu$ é maximizante.

Sejam $i$ o numero mais pequeno tal que $x_{i}=I, i^{-}<i$ o maior numero tal que $x_{i^{-}}<I_{1}$, e $i^{+}>i$ o menor numero tal que $x_{i^{+}}>I_{1}$, portanto $x_{j} \geq I_{1}$ para cada $j \in\left\{i^{-}+1, \ldots, i^{+}-1\right\}$, e $x_{j}=I$ para pelo menos um $j \in\left\{i^{-}+1, \ldots, i^{+}-1\right\}$.

Seja $\widetilde{\omega}=x_{i^{-}} y x_{i^{+}}\left(\right.$ou $\left.\widetilde{\omega}=x_{i^{-}} x_{i^{+}}\right)$onde $y$ tem só símbolos do alfabeto $F_{1}$. Observemos que $x=x_{0} \ldots x_{i^{-}-1} \omega x_{i^{+}+1} \ldots$ onde $\omega=x_{i^{-}} \ldots x_{i^{+}}$. Seja $m_{1}$ o comprimento do prefixo $x_{0} \ldots x_{i^{-}-1} \omega$.

Seja $\delta:=\min \left\{C_{1}, C_{2}\right\}-\left.\sup f\right|_{\cup_{a \geq I_{2}}[a]}>0$, dado que $\kappa_{m-1}(l, m \mid x)=\kappa(l, m \mid x)$, se substituímos $x_{l+m}$ por $\omega$, segue $S_{m+1} f\left(\sigma^{l} x\right)+\delta \leq S_{p} f(z)$.

Usando o mesmo procedimento substituindo $\omega$ por $\widetilde{\omega}$ obtemos $x^{1} \in \operatorname{Per}(\Sigma)$ com prefixo $\widetilde{m}_{1}$ tal que $S_{m_{1}} f(x)+\delta \leq S_{\widetilde{m}_{1}} f\left(x^{1}\right)$, repetindo o mesmo procedimento $k$-vezes começando na $k$-ésima aparição da palavra $\omega$ em $x$ e da mesma forma até a primeira aparição da palavra $\omega$ em $x$ obtemos $x^{k} \in \operatorname{Per}(\Sigma)$ satisfazendo $S_{m_{k}} f(x)+k \delta \leq S_{\widetilde{m}_{k}} f\left(x^{k}\right)$.

Seja $N$ tal que $(N-1) \delta \geq P_{0}|\min f|_{\Sigma_{F_{1}}} \mid+2 \bar{V}(f)$, então

$$
S_{\widetilde{m}_{N}} f\left(x^{N}\right) \geq S_{m_{N}} f(x)+\delta+P_{0}|\min f|_{\Sigma_{F_{1}}} \mid+2 \bar{V}(f)
$$

Dado que $\beta(x)=0$, aplicando o lema de Atkinson podemos escolher $l \geq m_{N}$ tal que $\left|S_{l} f(x)\right| \leq$ $\delta / 2$. Seja $m<l$ o maior numero tal que $x_{m}<I_{1}$, é claro que $m \geq m_{N}$ e dado que $x_{j} \leq I_{1}$ para

\footnotetext{
${ }^{3}$ É uma consequência direta do teorema de decomposição ergódica.
} 
$j \in\{m+1, \ldots, l\}$, e segue da escolha do $I_{1}$ que $S_{m} f(x) \geq S_{l} f(x)$, portanto

$$
S_{m} f(x) \geq-\delta / 2
$$

Seja $\widetilde{m}$ a posição de $x_{m}$ no ponto $x^{N} \in \operatorname{Per}(\Sigma)$, pela construção do $x^{N}$ temos $\sigma^{m_{N}} x=\sigma^{\widetilde{m}_{N}} x^{N}$, além disso $\widetilde{m}-\widetilde{m}_{N}=m-m_{N}$, então

$$
S_{\widetilde{m}} f\left(x^{N}\right)=S_{\widetilde{m}_{N}} f\left(x^{N}\right)+S_{m-m_{N}} f\left(\sigma^{m_{N}} x\right)
$$

portanto

$$
S_{\widetilde{m}} f\left(x^{N}\right) \geq S_{m} f(x)+\delta+P_{0}|\min f|_{\Sigma_{F_{1}}} \mid+2 \bar{V}(f) .
$$

Seja $\xi$ uma palavra ligando $x_{\widetilde{m}}^{N}$ ao $x_{0}^{N}$ composta unicamente de símbolos em $F_{1}$, e seja $z=$ $\overline{x_{0}^{N} \ldots x_{\tilde{m}}^{N} \xi}$, portanto o período de $z$ é $p=\widetilde{m}+|\xi|$ e

$$
S_{p} f(z) \geq S_{\widetilde{m}} f\left(x^{N}\right)-2 \bar{V}(f)-P_{0}|\min f|_{\Sigma_{F_{1}}} \mid \geq S_{m} f(x)+\delta \geq \delta / 2 .
$$

Em conclusão $\beta(z)=\frac{1}{p} S_{p} f(z) \geq \frac{\delta}{2 p}>0$ o qual é uma contradição.

\subsection{Limite em temperatura Zero para subshifts compactos}

Nesta seção vamos ver um teorema demonstrado por Leplaideur em [13], o teorema garante existência do limite em temperatura zero para potenciais localmente uniformemente constantes definidos em subshifts de Markov topologicamente transitivos com alfabeto finito, no entanto vamos ver parte da demonstração feita por Chazottes, Gambaudo e Ugalde em [7] que usa técnicas de renormalização para encontrar a caracterização da medida limite num caso onde o potencial satisfaz propriedades mais gerais do que o caso estudado por Leplaideur. O principal aporte nesses trabalhos foi dar uma forma explicita do limite em temperatura zero.

Ao longo desta seção vamos supor que o $\Sigma$ é um subshift de Markov com alfabeto $\mathcal{A}$ finito, e portanto é compacto.

Dizemos que $f$ é um potencial de Markov $^{4}$ se $f(x)=f\left(x_{0}, x_{1}\right)$ para todo $x \in \Sigma$, observe que neste caso $V_{n}(f)=0$ para cada $n \geq 2$. Sejam $f, g$ potenciais de Markov contínuos, dado que $\Sigma$ é um subshift de Markov compacto foi demonstrado em [11] que para cada $t>0$ o estado de equilíbrio $\mu_{t f+g}$ associado ao potencial $t f+g$ existe e é único.

\footnotetext{
${ }^{4}$ Esta propriedade é equivalente à propriedade do potencial ser localmente uniformemente constante em [12].
} 
Vamos definir $P:[0, \infty) \rightarrow \mathbb{R}$ como a função $t \mapsto P(t)=P_{G}(t f)$, observe que a função $P$ é convexa e portanto contínua.

Lema 11. [11] A função $P:(0, \infty) \rightarrow \mathbb{R}$ é diferenciável com $P^{\prime}(t)=\int f d \mu_{t f}$.

Seja $\beta$ como em (4.1) e $\bar{\Sigma} \subset \Sigma$ o menor conjunto compacto $\sigma$-invariante contendo o suporte de todas as medidas $f$-maximizantes, chamaremos esse conjunto de subshift maximizante.

Podemos assumir s.p.d.g. $\beta=P_{G}\left(\left.g\right|_{\bar{\Sigma}}\right)=0^{5}$. Seja $\bar{\Sigma}=\bigcup_{J=1}^{N} \bar{\Sigma}_{J}$ a decomposição do $\bar{\Sigma}$ em componentes transitivas, podemos ordenar essas componentes transitivas de modo que $P_{G}\left(\left.f\right|_{\bar{\Sigma}_{J}}\right)=0$ para cada $J \in\left\{1, \ldots, N_{f}\right\}$, e $P_{G}\left(\left.f\right|_{\Sigma_{J}}\right)<0$ para cada $J \in\left\{N_{f}+1, \ldots, N\right\}$, o anterior é consequência do lema 6 dado que no caso compacto

$$
P_{G}\left(\left.f\right|_{\bar{\Sigma}}\right)=\max \left\{P_{G}\left(\left.f\right|_{\bar{\Sigma}_{J}}\right): 1 \leq J \leq N\right\}
$$

Chamaremos $\bar{\Sigma}_{1}, \ldots, \bar{\Sigma}_{N_{f}}$ as componentes pesadas do subshift $\bar{\Sigma}$, além disso para cada $J \in$ $\left\{1, \ldots, N_{f}\right\}$ denotaremos por $\nu_{g, J}$ o único estado de equilíbrio associado ao potencial $\left.g\right|_{\bar{\Sigma}_{J}}$ e denotaremos por $\overline{\mathcal{A}}_{J}$ o alfabeto associado ao subshift $\bar{\Sigma}_{J}$. Vamos fazer o seguinte processo de renormalização do $\Sigma$.

Seja $\mathcal{A}^{\prime}=\left\{1,2, \ldots, N_{f}\right\}$, vamos definir um novo subshift de Markov $\Sigma^{\prime} \subset\left(\mathcal{A}^{\prime}\right)^{\mathbb{N}}$ com matriz de incidência $\mathbf{M}^{\prime}$ indexada em $\mathcal{A}^{\prime} \times \mathcal{A}^{\prime}$, onde

$$
\mathbf{M}_{J, K}^{\prime}=\left\{\begin{array}{l}
1, \text { se } \exists x \in \Sigma, \exists n \in \mathbb{N}: x_{0} \in \overline{\mathcal{A}}_{J}, x_{n} \in \overline{\mathcal{A}}_{K}, \text { e } \bigcup_{L=1}^{N_{f}}\left(\left\{x_{1}, \ldots, x_{n-1}\right\} \cap \overline{\mathcal{A}}_{L}\right)=\emptyset \\
0, \text { e.o.c. }
\end{array}\right.
$$

Dados quaisquer $a, c \in \mathcal{A}$ definimos o conjunto

$$
\operatorname{Path}(a, c)=\bigcup_{n \in \mathbb{N}}\left\{\omega \in \mathcal{A}^{n}: a_{0}=a, a_{n-1}=c, a_{i} \neq a_{j} \neq a, c \quad \forall 0<i<j<n-1\right\},
$$

e para cada $\omega=a_{0} \ldots a_{n-1} \in \operatorname{Path}(a, c)$ definimos $\bar{f}(\omega)=\sum_{i=0}^{n-2} f\left(a_{i}, a_{i+1}\right)$. Fixemos $c_{J} \in \overline{\mathcal{A}}_{J}$, e seja $a \in \overline{\mathcal{A}}_{J}$, dado que para todo $\omega \in \overline{\mathcal{A}}_{J}^{n}$ tal que $a_{0}=a_{n-1}$ temos $\bar{f}(\omega)=0$, então para toda palavra $\omega_{c_{J}, a}=c_{J} a_{1} \ldots a_{n-2} a$ começando em $c_{J}$ e finalizando em $a$ o valor $\bar{f}\left(\omega_{c_{J}, a}\right)$ é o mesmo, portanto $\vec{f}(a):=\bar{f}\left(\omega_{c_{J}, a}\right)$ está bem definido para cada $a \in \overline{\mathcal{A}}_{J}$.

Para $a \in \overline{\mathcal{A}}_{J}$ e $c \in \overline{\mathcal{A}}_{K}$ definimos o conjunto

$$
\widetilde{\operatorname{Path}}(a, c)=\left\{\omega \in \operatorname{Path}(a, c): \nexists i \operatorname{tq} \bar{f}\left(a_{i}, a_{i+1}\right)=0\right\},
$$

\footnotetext{
${ }^{5}$ No caso $f=g$ é uma consequência simples do lema 10 .
} 
e definimos $\vec{f}(a, c):=\max \{\bar{f}(\omega): \omega \in \widetilde{\operatorname{Path}}(a, c)\}$. Os potenciais renormalizados são definidos da seguinte forma

$$
\begin{aligned}
f^{\prime}(J, K) & :=\max _{a \in \overline{\mathcal{A}}_{J}, c \in \overline{\mathcal{A}}_{K}}\{\vec{f}(a)+\vec{f}(a, c)-\vec{f}(c)\} \\
g^{\prime}(J, K) & :=\log \left(\sum_{a, c \in \overline{\mathcal{A}}_{J, K}} v_{g, J}(a) w_{g, J}(c) \sum_{\omega \in \overline{\operatorname{Path}}(a, c)} \exp \left(\bar{f}(\omega)+P_{g}(\omega)\right)\right),
\end{aligned}
$$

onde $\overline{\mathcal{A}}_{J, K} \subset \overline{\mathcal{A}}_{J} \times \overline{\mathcal{A}}_{K}$ é o conjunto que maximiza $\vec{f}(a)+\vec{f}(a, c)-\vec{f}(c)$, o conjunto $\overline{\text { Path }}(a, c)$ está definido como

$$
\overline{\operatorname{Path}}(a, c)=\{\omega \in \operatorname{Path}(a, c): \bar{f}(\omega)=0\},
$$

$v_{g, J}, w_{g, J}$ são os autovetores associados ao autovalor $\lambda_{g, J}=\max \left|\operatorname{spec}\left(\overline{\mathbf{M}}_{g, J}\right)\right|$ onde a matriz $\overline{\mathbf{M}}_{g, J}$ é dada por ${ }^{6}$

$$
\overline{\mathbf{M}}_{g, J}= \begin{cases}\exp \left(g\left(x_{0}, x_{1}\right)\right) & , \text { se }\left[x_{0} x_{1}\right] \neq \emptyset \\ 0 & \text {, e.o.c. }\end{cases}
$$

e $P_{g}(\omega):=\log \left(\sum_{i=1}^{n-2} \sum_{k=0}^{\infty} \exp \left(k g\left(x_{i}, x_{i}\right)\right)\right)<\infty$.

O seguinte lema é o ponto chave da demonstração feita por Chazottes, Gambaudo e Ugalde.

Lema 12 (Renormalização). [7] Sejam $f, g: \Sigma \rightarrow \mathbb{R}$ potenciais de Markov, $\bar{\Sigma}$ o subshift $f$ maximizante, $\bar{\Sigma}=\bigcup_{J=1}^{N} \bar{\Sigma}_{J}$ sua decomposição em componentes transitivas e $f^{\prime}, g^{\prime}$ os potenciais renormalizados. Então existe $\delta(\mathcal{A}, f)$ tal que para $t$ suficientemente grande e todo cilindro $\left[b_{0} \ldots b_{n-1}\right]$ que satisfaz $\left[b_{0} \ldots b_{n-1}\right] \cap \bar{\Sigma}_{J} \neq \emptyset$ para algum $1 \leq J \leq N_{f}$, temos

$$
\mu_{t f+g}\left[b_{0} \ldots b_{n-1}\right]=\nu_{g, J}\left[b_{0} \ldots b_{n-1}\right] \mu_{t f^{\prime}+g^{\prime}}[J] \pm e^{-t \delta}
$$

O seguinte teorema da uma caracterização do limite em temperatura zero para os estados de equilíbrio quando o subshift é topologicamente transitivo e tem alfabeto finito, este resultado foi demonstrado primeiramente pelo Leplaideur em [13], no entanto depois disso foi dada outra demonstração por Chazottes, Gambaudo e Ugalde em [7]. As contas que aparecem na ultima parte deste capitulo são um rascunho da demonstração que aparece em [7] a qual é consequência do lema 12.

Teorema 9. [13][7] Sejam $\Sigma$ um subshift de Markov topologicamente transitivo e f,g potenciais

\footnotetext{
${ }^{6} \mathrm{~A}$ existência do autovalor $\lambda_{g, J}$ e dos autovetores $v_{g, J}, w_{g, J}$ é garantida pelo teorema de Perron-Frobenius.
} 
de Markov. Então

$$
\lim _{t \rightarrow \infty} \mu_{t f+g}=\sum_{J=1}^{N_{f}} \gamma_{J} \nu_{g, J}
$$

onde $0 \leq \gamma_{J} \leq 1$ e $\sum_{J=1}^{N_{f_{k}}} \gamma_{J}=1$. Além disso no caso $f=g$ a medida $\mu_{\infty}=\lim _{t \rightarrow \infty} \mu_{t f}$ satisfaz $\mu_{\infty} \in \mathcal{M}_{\max }(f)$.

Demonstração. A prova é feita em quatro passos:

I) Seja $b \notin \bigcup_{J=1}^{N_{f}} \overline{\mathcal{A}}_{J}$, dado que $\sum_{J=1}^{N_{f}} \mu_{t f^{\prime}+g^{\prime}}[J]=1$ e $\sum_{a \in \overline{\mathcal{A}}_{J}} \nu_{g, J}[a]=1$, pelo lema 12 temos para $t$ suficientemente grande

$$
\begin{aligned}
\mu_{t f+g}[b] & \leq 1-\mu_{t f+g}\left(\bigcup_{J=1}^{N_{f}} \overline{\mathcal{A}}_{J}\right) \\
& =1-\sum_{J=1}^{N_{f}} \sum_{a \in \overline{\mathcal{A}}_{J}} \mu_{t f+g}[a] \\
& \leq 1-\sum_{J=1}^{N_{f}}\left(\mu_{t f^{\prime}+g^{\prime}}[J]\left(\sum_{a \in \overline{\mathcal{A}}_{J}} \nu_{g, J}[a]\right)-e^{-t \delta}\right) \leq e^{-t \delta}|\mathcal{A}| .
\end{aligned}
$$

Seja $\left[b_{0} \ldots b_{n-1}\right]$ um cilindro satisfazendo $\left[b_{0} \ldots b_{n-1}\right] \cap \bar{\Sigma}_{J} \neq \emptyset$ para algun $J \in\left\{1, \ldots, N_{f}\right\}$ e $b_{i} \notin \bigcup_{J=1}^{N_{f}} \overline{\mathcal{A}}_{J}$ para ao menos um $b_{i}$. Dado que $\mu_{t f+g}$ é $\sigma$-invariante podemos assumir $b_{0}=b$, portanto $\left[b_{0} \ldots b_{n-1}\right] \subset[b]$ do qual segue $\mu_{t f+g}\left[b_{0} \ldots b_{n-1}\right] \leq \mu_{t f+g}[b]$. Dado que para cada $t>1$ é satisfeito $e^{-t \delta}<e^{-t \delta / 2}$, então para $t$ suficientemente grande $\mu_{t f+g}\left[b_{0} \ldots b_{n-1}\right] \leq e^{-t \delta / 2}$.

II) Pelo lema 12 temos para cada $J \in{\overline{\mathcal{A}^{\prime}}}_{K}$ e cada $K \in\left\{1, \ldots, N_{f^{\prime}}\right\}$

$$
\mu_{t f^{\prime}+g^{\prime}}[J]=\nu_{g^{\prime}, K}[J] \mu_{t f^{\prime \prime}+g^{\prime \prime}}[K] \pm e^{-t \delta^{\prime}}
$$

portanto segue do passo anterior que $\mu_{t f^{\prime}+g^{\prime}}[J] \leq e^{-t \delta^{\prime} / 2}$ para cada $J \notin \bigcup_{K=1}^{N_{f}^{\prime}} \overline{\mathcal{A}^{\prime}}{ }_{K}$ e $t$ suficientemente grande. Observe que $\nu_{g^{\prime}, K}[J]$ é o primeiro termo da fatoração do $\gamma_{J}$.

III) Seja $\left(\Sigma^{(i)}, t f^{(i)}+g^{(i)}\right)$ o sistema obtido depois do $i$-ésimo processo de renormalização, portanto $\mu_{t f^{(i)}+g^{(i)}}[K] \leq e^{-t \delta^{(i)} / 2}$. Temos duas possibilidades $\left|\mathcal{A}^{(i)}\right|<\left|\mathcal{A}^{(i-1)}\right|$ ou $\left|\mathcal{A}^{(i)}\right|=\left|\mathcal{A}^{(i-1)}\right|$.

Se $\left|\mathcal{A}^{(i)}\right|=\left|\mathcal{A}^{(i-1)}\right|$, então cada ponto periódico $\bar{J}=(J, J, \ldots)$ é uma componente pesada do shift $\Sigma^{(i-1)}$, portanto $[J J] \neq \emptyset$ para cada $J \in \mathcal{A}^{(i-1)}$ e usando (4.7) segue que $\mathbf{M}_{\bar{J}, \bar{J}}^{(i)}=0$ para todo $\bar{J} \in \mathcal{A}^{(i)}$, em consequência $\left|\mathcal{A}^{(i+1)}\right|<\left|\mathcal{A}^{(i)}\right|$.

O processo de renormalização finaliza quando achamos $m$ tal que $\mathcal{A}^{(m)}=\{1\}$, nesse caso 
temos $\mu_{t f^{(m)}+g^{(m)}}[1]=1$, portanto

$$
\mu_{t f+g}\left[b_{0} \ldots b_{n-1}\right]=\nu_{g, J}\left[b_{0} \ldots b_{n-1}\right] \underbrace{\nu_{g^{\prime}, K}[J] \ldots \nu_{g^{(m)}, 1}[L]}_{\gamma_{J}} \pm \sum_{J=0}^{m-1} e^{-t \delta^{(i)}} .
$$

Fazendo $t \rightarrow \infty$ obtemos $\lim _{t \rightarrow \infty} \mu_{t f+g}\left[b_{0} \ldots b_{n-1}\right]=\sum_{J=1}^{N_{f}} \gamma_{J} \nu_{g, J}\left[b_{0} \ldots b_{n-1}\right]$ para todo cilindro satisfazendo $\left[b_{0} \ldots b_{n-1}\right] \cap \bar{\Sigma}_{J} \neq \emptyset$ para algun $J \in\left\{1, \ldots, N_{f}\right\}$ e $b_{i} \notin \bigcup_{J=1}^{N_{f}} \overline{\mathcal{A}}_{J}$ para ao menos um $b_{i}$. Do anterior segue $\lim _{t \rightarrow \infty} \mu_{t f+g}=\sum_{J=1}^{N_{f}} \gamma_{J} \nu_{g, J}$.

IV) Vamos demonstrar que no caso $f=g$ a medida $\mu_{\infty}=\lim _{t \rightarrow \infty} \mu_{t f}$ satisfaz $\mu_{\infty} \in \mathcal{M}_{\text {max }}(f)^{7}$. Se $\mu_{\infty} \notin \mathcal{M}_{\text {max }}(f)$, então existe uma medida $\mu \in \mathcal{M}_{\max }(\Sigma)$ tal que $\int f d \mu-\int f d \mu_{\infty}>0$. Como $\Sigma$ tem alfabeto finito então o principio variacional implica $h(\mu)<\infty$. Definimos a função $l_{\mu}: \mathbb{R} \rightarrow \mathbb{R}$ como a função que a cada $t$ atribui o valor $l_{\mu}(t)=h(\mu)+t \int f d \mu$. Dado que a função $P(t)$ é convexa, então $P^{\prime}(t)$ é crescente do qual segue $\int f d \mu_{\infty} \geq \int f d \mu_{t f}=P^{\prime}(t)$. Portanto

$$
l_{\mu}^{\prime}(t)=\int f d \mu \geq \int f d \mu_{\infty}+\epsilon \geq P^{\prime}(t)+\epsilon .
$$

Então para $t$ suficientemente grande temos $l_{\mu}(t)>P(t)$, ou seja $h(\mu)+t \int f d \mu>P(t f)$ o qual contradiz o principio variacional. Em conclusão $\mu_{\infty} \in \mathcal{M}_{\max }(f)$.

\footnotetext{
${ }^{7}$ Dado que todo cilindro $\left[b_{0} \ldots b_{n-1}\right]$ é conjunto de continuidade para $\sum_{J=1}^{N_{f}} \gamma_{J} \nu_{g, J}$.
} 


\section{Capítulo 5}

\section{Limite em temperatura Zero para}

\section{subshifts não compactos}

Seja $\Sigma$ um subshift de Markov topologicamente transitivo com alfabeto enumerável e $f$ um potencial somável, com pressão finita e $\bar{V}(f)<\infty$. Neste capitulo vão aparecer as demonstrações dos resultados obtidos neste trabalho.

A ideia das demonstrações é fazer uma aproximação por subshifts compactos encaixados. Usando a existência de estados de equilíbrio no caso compacto, argumentos de rigidez e um manejo cuidadoso das entropias dos estados de equilíbrio associados às suas restrições compactas, vamos demonstrar existência do estado de equilíbrio associado a cada potencial $t f$ para todo $t>1$ e existência de pontos de acumulação quando $t \rightarrow \infty$.

Além disso quando o potencial $f$ é Markov, usando a caracterização feita em [13], vamos caracterizar o limite em temperatura zero quando o alfabeto é enumerável e vamos mostrar que essa medida limite é uma medida maximizante. Finalmente vamos demonstrar a continuidade da entropia com respeito ao parâmetro $t$ quando $t \rightarrow \infty$ e vamos mostrar que a entropia da medida limite é maximal, isso usando o resultado que aparece em [16].

Já foram feitos trabalhos na direção de subshifts de Markov com alfabeto enumerável, mas neles é requerido que o subshift seja finitamente primitivo ou que satisfaça a propriedade BIP $^{1}$. É importante ressaltar que estos são os primeiros resultados de limites em temperatura zero que vão além dessa propriedade.

Na primeira seção deste capítulo vamos construir uma sequência estritamente crescente de subshifts compactos encaixados que é a ferramenta principal na busca de demonstrar os resul-

\footnotetext{
${ }^{1}$ As duas propriedades sao equivalentes quando o subshift é topologicamente mixing.
} 
tados, além disso vamos mostrar que fixando qualquer valor de $t>1$ a sequência de estados de equilíbrio associados ao potencial restrito a cada um dos subshifts compactos é um conjunto justo.

Na segunda seção deste capítulo é onde vamos demonstrar a existência de estados de equilíbrio associados a cada potencial $t f$, isso usando a construção feita na primeira seção. Além disso vamos mostrar que essa sequência tem pontos de acumulação quando $t \rightarrow \infty$, demonstrando dessa forma o teorema A.

Na ultima seção deste capítulo demonstraremos os teoremas B e C, uma parte essencial nessas demonstrações são a caracterização feita em [13] e o teorema demonstrado em [16], também vai ser importante a construção feita nas primeiras duas seções.

\subsection{Aproximação por subshifts compactos}

Podemos supor s.p.d.g. que $f \leq 0$. Como o potencial $f$ é somável (2.4) e $\Sigma$ é topologicamente transitivo, pelo teorema 8 existe um conjunto finito $F \subset \mathcal{A}$ tal que

$$
\beta=\sup \left\{\int f d \nu: \nu \in \mathcal{M}_{\sigma}\left(\Sigma_{F}\right)\right\}
$$

onde $\Sigma_{F}$ é a restrição de $\Sigma$ ao alfabeto $F$. Dado que $\Sigma_{F}$ é compacto, então $\mathcal{M}_{\max }(f) \neq \emptyset$, além disso para cada $\mu \in \mathcal{M}_{\max }(f)$ é satisfeito $\operatorname{supp}(\mu) \subset \Sigma_{F}$.

Vamos construir uma sequência crescente $\left(\Sigma_{k}\right)_{k \in \mathbb{N}}$ de subshifts compactos de $\Sigma$ tais que para cada $k \in \mathbb{N}$ é satisfeito $\Sigma_{k} \subsetneq \Sigma_{k+1}$. Seja $\mathcal{A}_{k} \subsetneq \mathcal{A}$ o alfabeto associado ao $\Sigma_{k}$, e $f_{k}=\left.f\right|_{\Sigma_{k}}$ a restrição do $f$ ao $\Sigma_{k}$.

É suficiente escolher a sequência $\left(\mathcal{A}_{k}\right)_{k \in \mathbb{N}}$ da forma

$$
\mathcal{A}_{k}=\left\{0, \ldots, m_{k}\right\} \cup\left\{c \in \omega_{a b}:\left[a \omega_{a b} b\right] \neq \emptyset ; a, b \in\left\{0, \ldots, m_{k}\right\}\right\},
$$

onde $\omega_{a b}$ é uma palavra ${ }^{2}$ ligando $a$ e $b$, e $m_{k+1}=\max \left\{a: a \in \mathcal{A}_{k}\right\}+1$.

Seja

$$
\beta_{k}=\sup \left\{\int f_{k} d \nu: \nu \in \mathcal{M}_{\sigma}\left(\Sigma_{k}\right)\right\}
$$

Vamos definir $P:[1, \infty) \rightarrow \mathbb{R}$ como $P(t)=P_{G}(t f)<\infty$ e a sequência de funções $\left(P_{k}\right)_{k \in \mathbb{N}}$ com $P_{k}:[1, \infty) \rightarrow \mathbb{R}$ como $P_{k}(t)=P_{G}\left(t f_{k}\right)$. Claramente essas funções são convexas e portanto

\footnotetext{
${ }^{2} \mathrm{~A}$ palavra $\omega_{a b}$ existe porque $\Sigma$ é topologicamente transitivo.
} 
continuas, além disso dado que para cada $k \in \mathbb{N}$

$$
\sum_{\sigma^{n} x=x} \exp \left(S_{n} t f(x)\right) 1_{[a]}(x) \geq \sum_{\sigma^{n} x=x} \exp \left(S_{n} t f(x)\right) 1_{[a]}(x) 1_{\Sigma_{k}}(x),
$$

então temos $P_{k}(t) \leq P_{k+1}(t) \leq P(t)$. Além disso pelo lema 11 temos que cada uma das funções $\left(P_{k}\right)_{k \in \mathbb{N}}$ é analítica em $(1, \infty) \operatorname{com} P_{k}^{\prime}(t)=\int f_{k} d \mu_{t f_{k}}$, e segue do lema 6 que

$$
P(t)=\sup \left\{P_{k}(t): k \in \mathbb{N}\right\}=\lim _{k \rightarrow \infty} P_{k}(t)
$$

Vamos mostrar que a sequência $\left(\mu_{t f_{k}}\right)_{k \in \mathbb{N}} \subset \mathcal{M}_{\sigma}(\Sigma)$ tem uma subsequência convergente, mais ainda vamos ver que o limite da subsequência é um estado de equilíbrio associado ao potencial $t f$. Nessa direção vamos demonstrar primeiro que a sequência é justa.

Lema 13. Para cada $t>1$ a sequência $\left(\mu_{t f_{k}}\right)_{k \in \mathbb{N}} \subset \mathcal{M}_{\sigma}(\Sigma)$ é justa.

Demonstração. A demonstração é semelhante à demostração feita em [10].

Seja $\epsilon>0,\left(n_{m}\right)_{m \in \mathbb{N}} \subset \mathbb{N}$ uma sequência estritamente crescente e

$$
K=\left\{x \in \Sigma: 1 \leq x_{m} \leq n_{m} \text { para cada } m \in \mathbb{N}\right\}
$$

Portanto o conjunto $K$ é compacto na topologia produto, além disso

$$
\begin{aligned}
\mu_{t f_{k}}\left(K^{c}\right) & =\mu_{t f_{k}}\left(\bigcup_{m \in \mathbb{N}}\left\{x \in \Sigma: x_{m}>n_{m}\right\}\right) \\
& \leq \sum_{m \in \mathbb{N}} \mu_{t f_{k}}\left(\left\{x \in \Sigma: x_{m}>n_{m}\right\}\right) \\
& \leq \sum_{m \in \mathbb{N}} \sum_{a>n_{m}} \mu_{t f_{k}}\left(\left\{x \in \Sigma: x_{m}=a\right\}\right) \\
& =\sum_{m \in \mathbb{N}} \sum_{a>n_{m}} \mu_{t f_{k}}[a] .
\end{aligned}
$$

Para finalizar a demonstração do lema é suficiente escolher $\left(n_{m}\right)_{m \in \mathbb{N}} \subset \mathbb{N}$ tal que

$$
\sum_{a>n_{m}} \mu_{t f_{k}}[a]<\frac{\epsilon}{2^{m+1}}
$$

Seja $\eta=\frac{1}{p} \sum_{j=0}^{p-1} \delta_{\sigma^{j} \bar{x}}$ onde $\bar{x} \in \operatorname{Per}_{p}(\Sigma)$, então $\eta \in \mathcal{M}_{\sigma}(\Sigma)$ e além disso $S=\eta(f)$ satisfaz $-\infty<S<\infty$. Seja $S_{k}=\int f_{k} d \eta$, portanto $-\infty<S_{k}<\infty$ para cada $k \in \mathbb{N}$ e segue do principio 
variacional que

$$
P_{k}(t)-t S_{k}=P_{G}\left(t\left(f_{k}-S_{k}\right)\right) \geq h(\eta)+t\left(\int f_{k} d \eta-S_{k}\right)=h(\eta) \geq 0 .
$$

Dado que $\Sigma$ é topologicamente transitivo, existe uma constante $1 \leq C_{k}<\infty$ tal que

$$
\frac{\mu_{t f_{k}}\left[x_{0} \ldots x_{n-1}\right]}{\exp \left(t S_{n} f_{k}(x)-n P_{k}(t)\right)}=C_{k}^{ \pm 1}
$$

onde $C_{k}=\exp \left(4 \bar{V}\left(t f_{k}\right)\right) \leq \exp (4 \bar{V}(t f))=\exp (4 t \bar{V}(f))<\infty$.

Seja $S^{\inf }=\inf \left\{S_{k}: k \in \mathbb{N}\right\}$, como a medida $\eta$ é periódica, então $S^{\text {inf }}>-\infty$, portanto para cada $x \in[a]$

$$
\begin{aligned}
\mu_{t f_{k}}[a] & \leq \exp \left(4 t \bar{V}(f)+t f_{k}(x)-P_{k}(t)\right) \\
& \leq \exp \left(4 t \bar{V}(f)+\left.t \sup f\right|_{[a]}-P_{k}(t)\right) \\
& =\exp \left(t\left(4 \bar{V}(f)+\left.\sup f\right|_{[a]}-S_{k}\right)\right) \exp \left(t S_{k}-P_{k}(t)\right) \\
& \leq \exp \left(t\left(4 \bar{V}(f)+\left.\sup f\right|_{[a]}-S^{\inf }\right)\right) .
\end{aligned}
$$

Dado que $f$ é coercivo, então para a suficientemente grande $4 \bar{V}(f)+\left.\sup f\right|_{[a]}-S^{\text {inf }} \leq 0$, e dado que $t>1$ segue

$$
\mu_{t f_{k}}[a] \leq \exp \left(4 \bar{V}(f)+\left.\sup f\right|_{[a]}-S^{\inf }\right),
$$

além disso como o $f$ é somável podemos escolher a sequência $\left(n_{m}\right)_{m \in \mathbb{N}}$ satisfazendo a seguinte desigualdade

$$
\sum_{a>n_{m}} \exp \left(\left.\sup f\right|_{[a]}\right)<\frac{\epsilon}{2^{m+1}} \exp \left(S^{\inf }-4 \bar{V}(f)\right) .
$$

Portanto para cada $k \in \mathbb{N}$

$$
\sum_{a>n_{m}} \mu_{t f_{k}}[a] \leq \sum_{a>n_{m}} \exp \left(4 \bar{V}(f)+\left.\sup f\right|_{[a]}-S^{\inf }\right)<\frac{\epsilon}{2^{m+1}}
$$

Usando (5.2) e (5.4) concluímos

$$
\mu_{t f_{k}}\left(K^{c}\right)<\sum_{m \in \mathbb{N}} \frac{\epsilon}{2^{m+1}}=\epsilon
$$

Dado que a sequência $\left(\mu_{t f_{k}}\right)_{k \in \mathbb{N}} \subset \mathcal{M}_{\sigma}(\Sigma)$ é justa, pelo teorema 1 existem uma sequência 
$\left(\mu_{t f_{k_{m}}}\right)_{m \in \mathbb{N}} \subset\left(\mu_{t f_{k}}\right)_{k \in \mathbb{N}}$ e uma medida $\mu_{t} \in \mathcal{M}_{\sigma}(\Sigma)$ tal que $\lim _{m \rightarrow \infty} \mu_{t f_{k_{m}}}=\mu_{t}$. Nosso objetivo é provar que $\mu_{t}$ é de fato um estado de equilíbrio associado ao potencial $t f$, ou seja $\mu_{t}=\mu_{t f}$.

\subsection{Demonstração do teorema A}

Dado que $\mu_{t f_{k_{m}}}$ é estado de equilíbrio para cada $m \in \mathbb{N}$, então

$$
P_{k_{m}}(t)=h\left(\mu_{t f_{k_{m}}}\right)+\int t f_{k_{m}} d \mu_{t f_{k_{m}}}
$$

e dado que a sequência $\left(P_{k_{m}}(t)\right)_{m \in \mathbb{N}}$ é crescente, então segue do lema 6 que

$$
\lim _{m \rightarrow \infty} P_{k_{m}}(t)=P(t)
$$

Observemos que para cada $m \in \mathbb{N}$ temos

$$
\mu_{t f_{k_{m}}}[a] \leq \exp \left(4 t \bar{V}(f)+\sup \left(\left.t f\right|_{[a]}\right)-P_{0}(t)\right)
$$

Além disso $\mu_{t}(\partial[a])=0$ para cada $a \in \mathcal{A}$, ou seja o cilindro $[a]$ é um conjunto de continuidade da medida $\mu_{t}$, então fazendo $m \rightarrow \infty$ nós conseguirmos a seguinte desigualdade

$$
\mu_{t}[a] \leq \exp \left(4 t \bar{V}(f)+\sup \left(\left.t f\right|_{[a]}\right)-P_{0}(t)\right)
$$

portanto

$$
\begin{aligned}
\int-t f d \mu_{t} & =\int \sum_{a \in \mathcal{A}}-\left.t f\right|_{[a]} d \mu_{t} \\
& =\sum_{a \in \mathcal{A}} \int-\left.t f\right|_{[a]} d \mu_{t} \\
& \leq \sum_{a \in \mathcal{A}} \sup \left(-\left.t f\right|_{[a]}\right) \mu_{t}[a] \\
& \leq \sum_{a \in \mathcal{A}} \sup \left(-\left.t f\right|_{[a]}\right) \exp \left(4 t \bar{V}(f)+\sup \left(\left.t f\right|_{[a]}\right)-P_{0}(t)\right) \\
& =C_{t} \sum_{a \in \mathcal{A}} \sup \left(-\left.t f\right|_{[a]}\right) \exp \left(\sup \left(\left.t f\right|_{[a]}\right)\right)<\infty
\end{aligned}
$$

onde $C_{t}=\exp \left(4 t \bar{V}(f)-P_{0}(t)\right)$. Fazendo um procedimento similar podemos mostrar que para 
todo $m \in \mathbb{N}$

$$
\int-t f d \mu_{t f_{k_{m}}} \leq C_{t} \sum_{a \in \mathcal{A}} \sup \left(-\left.t f\right|_{[a]}\right) \exp \left(\sup \left(\left.t f\right|_{[a]}\right)\right)<\infty
$$

A desigualdade acima mostra que a sequência $\left(\int-t f d \mu_{t f_{k_{m}}}\right)_{m \in \mathbb{N}}$ é limitada por acima o qual é essencial para a demonstração do seguinte lema.

Lema 14. Para cada $t>1$ é satisfeito $\int t f d \mu_{t}=\lim _{m \rightarrow \infty} \int t f d \mu_{t f_{k_{m}}}$.

Demonstração. Observe que a demonstração deste lema não é uma consequência imediata das propriedades da topologia fraca* dado que o potencial $t f$ não é limitado (2.4), mas vamos fazer uma aproximação dele por potenciais limitados, e usando as desigualdades (5.5) e (5.6) vamos obter o resultado.

Para cada $n \in \mathbb{N}$, vamos definir a função $f^{(n)}$ como

$$
f^{(n)}(x)= \begin{cases}f(x) & , \text { se } f(x) \geq-n \\ 0 & \text { e.o.c. }\end{cases}
$$

Observe que a função $f^{(n)}$ é continua limitada, portanto para cada $n \in \mathbb{N}$ temos

$$
\lim _{m \rightarrow \infty} \int t f^{(n)} d \mu_{t f_{k_{m}}}=\int t f^{(n)} d \mu_{t}
$$

Seja $g^{(n)}=f-f^{(n)}$, então $g^{(n)} \leq 0$ e para cada $x \in \Sigma$ temos $\left|g^{(n)}(x)\right| \leq|f(x)|$.

Seja $\epsilon>0$, queremos mostrar que existe $n_{0} \in \mathbb{N}$ tal que para todo $n \geq n_{0}$ e todo $m \in \mathbb{N}$ é satisfeito $\left|\int t g^{(n)} d \mu_{t f_{k_{m}}}\right|<\frac{\epsilon}{4}$ e $\left|\int t g^{(n)} d \mu_{t}\right|<\frac{\epsilon}{4}$.

Com efeito, pelo lema 3 , existe $n^{\prime} \in \mathbb{N}$ tal que

$$
\sum_{a>n^{\prime}} \sup \left(-\left.t f\right|_{[a]}\right) \exp \left(4 t \bar{V}(f)+\sup \left(\left.t f\right|_{[a]}\right)-P_{0}(t)\right)<\frac{\epsilon}{4}
$$

portanto escolhendo $n_{0}$ suficientemente grande, tal que $\left|g_{\left(n_{0}\right)}(x)\right|=0$ para cada $x \in \bigcup_{a=0}^{n^{\prime}-1}[a]$, 
então para cada $n \geq n_{0}$ é satisfeito

$$
\begin{aligned}
\left|\int t g^{(n)} d \mu_{t}\right| & \leq \int\left|t g^{(n)}\right| d \mu_{t} \\
& =\int_{\bigcup_{a>n^{\prime}}[a]}\left|t g^{(n)}\right| d \mu_{t} \\
& \leq \int_{\bigcup_{a>n^{\prime}}[a]}-t f d \mu_{t} \\
& \leq \sum_{a>n^{\prime}} \sup \left(-\left.t f\right|_{[a]}\right) \mu_{t}[a] \\
& \leq \sum_{a>n^{\prime}} \sup \left(-\left.t f\right|_{[a]}\right) \exp \left(4 t \bar{V}(f)+\sup \left(\left.t f\right|_{[a]}\right)-P_{0}(t)\right) \\
& <\frac{\epsilon}{4}
\end{aligned}
$$

Fazendo o mesmo procedimento podemos demonstrar que

$$
\left|\int t g^{(n)} d \mu_{t f_{k m}}\right|<\frac{\epsilon}{4}
$$

Além disso, dado que $\mu_{t}=\lim _{m \rightarrow \infty} \mu_{t f_{k_{m}}}$, então fixando $n \geq n_{0}$, temos que existe $m_{0}$ tal que para cada $m \geq m_{0}$

$$
\left|\int t f^{(n)} d \mu_{t f_{k m}}-\int t f^{(n)} d \mu_{t}\right|<\frac{\epsilon}{2}
$$

portanto

$$
\begin{aligned}
\left|\int t f d \mu_{t f_{k_{m}}}-\int t f d \mu_{t}\right| & \leq\left|\int t f^{(n)} d \mu_{t f_{k_{m}}}-\int t f^{(n)} d \mu_{t}\right|+\left|\int t g^{(n)} d \mu_{t f_{k_{m}}}\right|+\left|\int t g^{(n)} d \mu_{t}\right| \\
& <\epsilon
\end{aligned}
$$

Observe que para cada $m \in \mathbb{N}$ temos

$$
h\left(\mu_{t f_{k_{m}}}\right)=P_{k_{m}}(t)-\int t f_{k_{m}} d \mu_{t f_{k_{m}}}<\infty
$$

Além disso $P_{k_{m}}(t) \leq P(t)$ para cada $m \in \mathbb{N}$ e por (5.6) a sequência $\left(\int-t f_{k_{m}} d \mu_{t f_{k_{m}}}\right)_{m \in \mathbb{N}}$ é limitada por acima, então existe uma constante $D_{t}>0$ tal que para todo $m \in \mathbb{N}$

$$
h\left(\mu_{t f_{k_{m}}}\right) \leq D_{t}
$$


Dado que $\alpha=\{[a]: a \in \mathcal{A}\}$ é uma partição geradora para o $\Sigma$, então para toda $\mu \in \mathcal{M}_{\sigma}(\Sigma)$ é satisfeito

$$
h(\mu)=\inf _{n \geq 1} \frac{1}{n} H\left(\mu \mid \alpha^{n}\right) .
$$

Portanto temos o seguinte resultado.

Proposição 2. Para cada $N \in \mathbb{N}$ existe $n_{0} \geq N$ tal que a sequência $\left(H\left(\mu_{t f_{k_{m}}} \mid \alpha^{n_{0}}\right)\right)_{m \in \mathbb{N}}$ é limitada.

Demonstração. Suponha que a afirmação é falsa, ou seja existe algum $N_{0} \in \mathbb{N}$ tal que para qualquer $n \geq N_{0}$ a sequência $\left(H\left(\mu_{t f_{k_{m}}} \mid \alpha^{n}\right)\right)_{m \in \mathbb{N}}$ não é limitada. Sejam $n^{\prime} \geq N_{0}$ e $B^{\prime}>0$, então existe uma sequência $\left(k_{l}\right)_{l \in \mathbb{N}} \subset\left(k_{m}\right)_{m \in \mathbb{N}}$ tal que $H\left(\mu_{t f_{k_{l}}} \mid \alpha^{n^{\prime}}\right)>B^{\prime}$ para todo $l \in \mathbb{N}$, particularmente temos que $\frac{1}{n^{\prime}} H\left(\mu_{t f_{k_{l}}} \mid \alpha^{n^{\prime}}\right)>\frac{B^{\prime}}{n^{\prime}}$. Dado que $B^{\prime}$ foi escolhido de forma arbitraria, então é possível escolher uma sequência $\left(k_{l}\right)_{l \in \mathbb{N}}$ tal que a ultima desigualdade é verdadeira para o valor $B^{\prime}=2 B n^{\prime}$, ou seja $\frac{1}{n^{\prime}} H\left(\mu_{t f_{k_{l}}} \mid \alpha^{n^{\prime}}\right)>2 B$ para todo $l \in \mathbb{N}$. Porém a sequência $\left(h\left(\mu_{t f_{k_{m}}}\right)\right)_{m \in \mathbb{N}}$ é limitada superiormente com cota superior $B$, então pela definição de entropia de Kolmogorov-Sinai obtemos uma contradição .

Dado que $\Sigma$ não é compacto, então a partição natural do $\Sigma$ não é finita, portanto a entropia associada a essa partição não é necessariamente contínua e portanto não podemos garantir que a entropia seja semicontínua superiormente. O seguinte lema ajuda a solucionar esse problema.

Lema 15. Seja $\mu_{t}=\lim _{m \rightarrow \infty} \mu_{t f_{k_{m}}}$, então para cada $N \in \mathbb{N}$ existem $n_{0} \geq N$ e uma subsequência $\left(\mu_{t f_{k_{l}}}\right)_{l \in \mathbb{N}} \subset\left(\mu_{t f_{k_{m}}}\right)_{m \in \mathbb{N}}$ tal que

$$
\lim _{l \rightarrow \infty} H\left(\mu_{t f_{k_{l}}} \mid \alpha^{n_{0}}\right)=H\left(\mu_{t} \mid \alpha^{n_{0}}\right)
$$

Demonstração. Seja $N \in \mathbb{N}$, pela proposição 2 existe $n_{0} \geq N$ tal que $\left(H\left(\mu_{t f_{k_{m}}} \mid \alpha^{n_{0}}\right)\right)_{m \in \mathbb{N}}$ é limitada superiormente, portanto existe $\left(k_{l}\right)_{l \in \mathbb{N}} \subset\left(k_{m}\right)_{m \in \mathbb{N}}$ tal que $\lim _{l \rightarrow \infty} H\left(\mu_{t f_{k_{l}}} \mid \alpha^{n_{0}}\right)<\infty$.

Mais ainda, existe $l_{0} \in \mathbb{N}$ tal que para todo $j \geq l_{0}$ temos que

$$
\inf _{l \geq l_{0}}\left\{-\mu_{t f_{k_{l}}}[\omega] \log \left(\mu_{t f_{k_{l}}}[\omega]\right)\right\} \leq-\mu_{t f_{k_{j}}}[\omega] \log \left(\mu_{t f_{k_{j}}}[\omega]\right)
$$

portanto somando sobre todos os $[\omega] \in \alpha^{n_{0}}$ segue

$$
\sum_{[\omega] \in \alpha^{n_{0}}} \inf _{l \geq l_{0}}\left\{-\mu_{t f_{k_{l}}}[\omega] \log \left(\mu_{t f_{k_{l}}}[\omega]\right)\right\} \leq \inf _{j \geq l_{0}}\left\{\sum_{[\omega] \in \alpha^{n_{0}}}-\mu_{t f_{k_{j}}}[\omega] \log \left(\mu_{t f_{k_{j}}}[\omega]\right)\right\}
$$


Mais ainda, tomando o limite quando $l_{0} \rightarrow \infty$ e usando o teorema de convergência monótona pra a função $\phi_{m}([\omega])=\inf _{l \geq m}\left\{-\mu_{t f_{k_{l}}}[\omega] \log \left(\mu_{t f_{k_{l}}}[\omega]\right)\right\}$ com a medida de contagem sobre o conjunto $\left\{[\omega]:[\omega] \in \alpha^{n_{0}}\right\}$, obtemos o seguinte

$$
\sum_{[\omega] \in \alpha^{n_{0}}} \liminf _{l \rightarrow \infty}\left(-\mu_{t f_{k_{l}}}[\omega] \log \left(\mu_{t f_{k_{l}}}[\omega]\right)\right) \leq \liminf _{l \rightarrow \infty} \sum_{[\omega] \in \alpha^{n_{0}}}-\mu_{t f_{k_{l}}}[\omega] \log \left(\mu_{t f_{k_{l}}}[\omega]\right)
$$

Além disso, dado que $\mu_{t}=\lim _{l \rightarrow \infty} \mu_{t f_{k_{l}}}$ e $\mu_{t}(\partial[\omega])=0$ para cada cilindro $[\omega] \in \alpha^{n_{0}}$, então temos que $\mu_{t}[\omega] \log \left(\mu_{t}[\omega]\right)=\lim _{l \rightarrow \infty} \mu_{t f_{k_{l}}}[\omega] \log \left(\mu_{t f_{k_{l}}}[\omega]\right)$.

Portanto

$$
\sum_{[\omega] \in \alpha^{n_{0}}}-\mu_{t}[\omega] \log \left(\mu_{t}[\omega]\right) \leq \liminf _{l \rightarrow \infty} \sum_{[\omega] \in \alpha^{n_{0}}}-\mu_{t f_{k_{l}}}[\omega] \log \left(\mu_{t f_{k_{l}}}[\omega]\right)
$$

Usando o mesmo procedimento trocando os ínfimos por supremos e mudando as desigualdades podemos demonstrar que

$$
\sum_{[\omega] \in \alpha^{n_{0}}}-\mu_{t}[\omega] \log \left(\mu_{t}[\omega]\right) \geq \limsup _{l \rightarrow \infty} \sum_{[\omega] \in \alpha^{n_{0}}}-\mu_{t f_{k_{l}}}[\omega] \log \left(\mu_{t f_{k_{l}}}[\omega]\right)
$$

portanto obtemos a seguinte igualdade

$$
H\left(\mu_{t} \mid \alpha^{n_{0}}\right)=\lim _{l \rightarrow \infty} H\left(\mu_{t f_{k_{l}}} \mid \alpha^{n_{0}}\right)
$$

O seguinte lema é o último passo para garantir que a medida $\mu_{t}$ é um estado de equilíbrio associado ao potencial $t f$ para cada $t>1$, ele mostra que podemos encontrar uma sequência $\left(\mu_{t f_{k_{l}}}\right)_{l \in \mathbb{N}}$ tal que a entropia é semicontínua superiormente quando $l \rightarrow \infty$.

Lema 16. Seja $\left(\mu_{t f_{k_{l}}}\right)_{l \in \mathbb{N}}$ uma sequência obtida pela construção do lema 15, então lim $\sup _{l \rightarrow \infty} h\left(\mu_{t f_{k_{l}}}\right) \leq$ $h\left(\mu_{t}\right)$.

Demonstração. Suponha que o lema é falso, ou seja $\lim \sup _{l \rightarrow \infty} h\left(\mu_{t f_{k_{l}}}\right)>h\left(\mu_{t}\right)$, então podemos escolher $\epsilon>0$ tal que $h\left(\mu_{t}\right) \leq \lim \sup _{l \rightarrow \infty} h\left(\mu_{t f_{k_{l}}}\right)-3 \epsilon$. Segue da definição de entropia de Kolmogorov-Sinai $(2.2)$ temos que $\frac{1}{n} H\left(\mu_{t} \mid \alpha^{n}\right) \leq h\left(\mu_{t}\right)+\epsilon$ para valores de $n$ suficientemente grandes, e pelo lema 15 existe $n_{0} \geq n$ tal que $\lim _{l \rightarrow \infty} H\left(\mu_{t f_{k_{l}}} \mid \alpha^{n_{0}}\right)=H\left(\mu_{t} \mid \alpha^{n_{0}}\right)$. Finalmente usando novamente a definição $(2.2)$ segue que $h\left(\mu_{t f_{k_{j}}}\right) \leq \frac{1}{n_{0}} H\left(\mu_{t f_{k_{j}}} \mid \alpha^{n_{0}}\right)$, portanto para $l_{0} \in \mathbb{N}$ 
suficientemente grande temos que

$$
\begin{aligned}
h\left(\mu_{t f_{k_{j}}}\right) & \leq \frac{1}{n_{0}} H\left(\mu_{t} \mid \alpha^{n_{0}}\right)+\epsilon \\
& \leq h\left(\mu_{t}\right)+2 \epsilon \\
& \leq \limsup _{l \rightarrow \infty} h\left(\mu_{t f_{k_{l}}}\right)-\epsilon,
\end{aligned}
$$

então tomando limsup quando $j \rightarrow \infty$, obtemos uma contradição. Portanto podemos concluir que $\lim \sup _{l \rightarrow \infty} h\left(\mu_{t f_{k_{l}}}\right) \leq h\left(\mu_{t}\right)$.

A demonstração do teorema A é uma consequência da construção feita ao longo desta seção. Observe que para garantir a existência do estado de equilíbrio associado ao potencial $t f$ não são usadas as propriedades do operador de Ruelle $L_{t f}$, portanto o abordagem usado na demonstração é completamente diferente ao abordagem usual.

Demonstração. (Teorema A)

Seja $t>1$ e $\left(\mu_{t f_{k_{l}}}\right)_{l \in \mathbb{N}}$ uma sequência obtida no lema 15 , então $\lim _{l \rightarrow \infty} P_{k_{l}}(t)=P(t)$ e $\lim _{l \rightarrow \infty} \int t f d \mu_{t f_{k_{l}}}=\int t f d \mu_{t}$, portanto

$$
P(t)=\limsup _{l \rightarrow \infty} P_{k_{l}}(t) \leq \limsup _{l \rightarrow \infty} h\left(\mu_{t f_{k_{l}}}\right)+\limsup _{l \rightarrow \infty} \int t f_{k_{l}} d \mu_{t f_{k_{l}}} \leq h\left(\mu_{t}\right)+\int t f d \mu_{t} .
$$

A igualdade acima mostra que $\mu_{t}$ é um estado de equilíbrio associado ao potencial $t f$. e dado que $P(t f)<\infty$ e $\bar{V}(t f)=\bar{V}(t f)<\infty$, então o estado de equilíbrio é único, ou seja $\mu_{t}=\mu_{t f}$.

Para demonstrar que a sequência $\left(\mu_{t f}\right)_{t>1}$ tem um ponto de acumulação quando $t \rightarrow \infty$, observe que a parte direita da desigualdade (5.3) não depende do $t$, então tomando o limite quando $k \rightarrow \infty$ obtemos que para cada $t>1$ é satisfeito

$$
\mu_{t f}[a] \leq \exp \left(4 \bar{V}(f)+\left.\sup f\right|_{[a]}-S^{\inf }\right)
$$

Fazendo o mesmo procedimento do lema 13 podemos concluir que a sequência $\left(\mu_{t f}\right)_{t>1}$ é justa. Tomando uma subsequência $t_{k} \rightarrow \infty$ podemos encontrar um ponto de acumulação da sequência $\left(\mu_{t f}\right)_{t>1}$ no infinito, portanto o teorema A é demonstrado.

Observe que na demonstração do teorema A, a construção da sequência $\left(\mu_{t f_{k_{l}}}\right)_{l \in \mathbb{N}}$ que converge para $\mu_{t}$ quando $l \rightarrow \infty$ parece um pouco artificial. O seguinte corolário mostra que de fato a sequência toda $\left(\mu_{t f_{k}}\right)_{k \in \mathbb{N}}$ também converge para a mesma medida. 
Corolário 1. Para cada $t>1$ a sequência de estados de equilíbrio $\left(\mu_{t f_{k}}\right)_{k \in \mathbb{N}}$ converge para $\mu_{t f}$.

Demonstração. Suponhamos que a sequência $\left(\mu_{t f_{k}}\right)_{k \in \mathbb{N}}$ não é convergente. Dado que $\mathcal{M}_{\sigma}(\Sigma)$ é metrizável com a distância $d$. Então, existe $\epsilon_{0}>0$ e uma subsequência $\left(\mu_{t f_{k_{j}}}\right)_{j \in \mathbb{N}} \subset\left(\mu_{t f_{k}}\right)_{k \in \mathbb{N}}$ tal que para todo $j \in \mathbb{N}$

$$
d\left(\mu_{t f_{k_{j}}}, \mu_{t f}\right) \geq \epsilon_{0}
$$

Em particular, a sequência $\left(\mu_{t f_{k_{j}}}\right)_{j \in \mathbb{N}}$ não converge para $\mu_{t f}$.

Agora pelo lema 13 a sequência $\left(\mu_{t f_{k_{j}}}\right)_{j \in \mathbb{N}}$ é justa, então usando o mesmo argumento da demonstração do teorema A, existe $\left(\mu_{t f_{k_{i}}}\right)_{i \in \mathbb{N}} \subset\left(\mu_{t f_{k_{j}}}\right)_{j \in \mathbb{N}}$ tal que

$$
\lim _{i \rightarrow \infty} \mu_{t f_{k_{i}}}=\mu_{t f}
$$

porém o estado de equilíbrio é único, portanto obtemos uma contradição.

\subsection{Demonstração dos teoremas B e C}

As ferramentas clave na demonstração destes resultados são a caracterização do limite em temperatura zero feita em [13] e o resultado de existência de medidas maximizantes em [3].

Proposição 3. Existe $k_{0}$ tal que para cada $k \geq k_{0}$ é satisfeito $\beta_{k}=\beta$, e $\mathcal{M}_{\max }\left(f_{k}\right)=\mathcal{M}_{\max }(f)$.

Demonstração. Seja $F \subset \mathbb{N}$ como no teorema 8, portanto toda medida $\mu \in \mathcal{M}_{\max }(f)$ satisfaz $\operatorname{supp}(\mu) \subset \Sigma_{F}$. além disso segue da construção dos $\Sigma_{k}$ 's que existe $k_{0} \geq 0$ tal que $\Sigma_{F} \subset \Sigma_{k_{0}}$, portanto podemos supor $\Sigma_{F}=\Sigma_{k_{0}}$, então

$$
\begin{aligned}
\beta & =\sup \left\{\int f d \nu: \nu \in \mathcal{M}_{\sigma}\left(\Sigma_{k_{0}}\right)\right\} \\
& =\sup \left\{\int f_{k_{0}} d \nu: \nu \in \mathcal{M}_{\sigma}\left(\Sigma_{k_{0}}\right)\right\} \\
& =\beta_{k_{0}} .
\end{aligned}
$$

Observe que para cada $k$ temos $\beta_{k} \leq \beta_{k+1} \leq \beta^{3}$, então $\beta_{k}=\beta$ para cada $k \geq k_{0}$.

Segue da igualdade anterior que para toda $\mu \in \mathcal{M}_{\max }(f)$ é satisfeito $\operatorname{supp}(\mu) \subset \Sigma_{k_{0}}$, portanto

$$
\beta=\int f d \mu=\int f_{k} d \mu
$$

\footnotetext{
${ }^{3} \mathcal{M}_{\sigma}\left(\Sigma_{k}\right) \subset \mathcal{M}_{\sigma}\left(\Sigma_{k+1}\right)$, além disso $\mu\left(f_{k}\right)=\mu\left(f_{k+1}\right)$ para cada $\mu \in \mathcal{M}_{\sigma}\left(\Sigma_{k}\right)$.
} 
ou seja $\mu \in \mathcal{M}_{\max }\left(f_{k}\right)$ para todo $k \geq k_{0}$.

Para demonstrar a outra desigualdade vamos supor $k \geq k_{0}$ e $\mu_{k} \in \mathcal{M}_{\max }\left(f_{k}\right)$, dado que $\operatorname{supp}\left(\mu_{k}\right) \subset \Sigma_{k}$, então

$$
\beta_{k}=\int f_{k} d \mu_{k}=\int f d \mu_{k}
$$

e dado que $\beta_{k}=\beta$, então $\mu_{k} \in \mathcal{M}_{\max }(f)$.

No seguinte lema obtemos uma caracterização do limite em temperatura zero para cada subshift compacto usando a caracterização feita em [13] e demonstramos que o limite em temperatura zero coincide para todo $k \geq k_{0}$.

É importante ressaltar que a hipótese do potencial $f$ ser Markov é necessária para garantir a existência do limite, em [8] é dado um exemplo no qual o limite não existe para um potencial Lipschitz.

De aqui para o frente sempre vamos assumir que o potencial $f$ é de Markov, ou seja $f(x)=$ $f\left(x_{0}, x_{1}\right)$.

Lema 17. Existe uma medida $\mu_{\infty} \in \mathcal{M}_{\sigma}(\Sigma)$ tal que $\mu_{\infty}=\lim _{t \rightarrow \infty} \mu_{t f_{k}}$ para cada $k \geq k_{0}$, além disso $\mu_{\infty} \in \mathcal{M}_{\max }(f)$.

Demonstração. Para cada $k$ seja $\bar{\Sigma}_{k}$ o subshift maximizante do $\Sigma_{k}$. No capítulo 4 vimos que cada um dos $\bar{\Sigma}_{k}$ 's admite uma decomposição em componentes transitivas

$$
\bar{\Sigma}_{k}=\bigcup_{J=1}^{N_{k}} \bar{\Sigma}_{k, J} .
$$

além disso podemos ordenar essas componentes de modo que $P_{G}\left(\left.f_{k}\right|_{\bar{\Sigma}_{k, J}}\right)=P_{G}\left(\left.f_{k}\right|_{\bar{\Sigma}_{k}}\right)$ para cada $J \in\left\{1, \ldots, N_{f_{k}}\right\}$ e $P_{G}\left(\left.f_{k}\right|_{\bar{\Sigma}_{k, J}}\right)<P_{G}\left(\left.f_{k}\right|_{\bar{\Sigma}_{k}}\right)$ para cada $J \in\left\{N_{f_{k}}+1, \ldots, N_{k}\right\}$. Seja $\nu_{k, J}$ o único estado de equilíbrio associado ao potencial $\left.f_{k}\right|_{\bar{\Sigma}_{k, J}}$, segue do teorema 9 que $\lim _{t \rightarrow \infty} \mu_{t f_{k}}=$ $\sum_{J=1}^{N_{f_{k}}} \gamma_{J} \nu_{k, J}$, onde $0 \leq \gamma_{J} \leq 1, \sum_{J=1}^{N_{f_{k}}} \gamma_{J}=1$, e cada um dos $\gamma_{J}$ 's pode ser calculado usando um algoritmo de renormalização, ou seja para toda função continua limitada $g: \Sigma \rightarrow \mathbb{R}$

$$
\lim _{t \rightarrow \infty} \int g d \mu_{t f_{k}}=\sum_{J=1}^{N_{f_{k}}} \gamma_{J} \int g d \nu_{k, J}
$$

além disso pela ultima parte do teorema 9 temos $\sum_{J=1}^{N_{f_{k}}} \gamma_{J} \nu_{k, J} \in \mathcal{M}_{\max }\left(f_{k}\right)$. Se $k \geq k_{0}$ segue da proposição 3 que $\mathcal{M}_{\max }\left(f_{k}\right)=\mathcal{M}_{\max }(f)$, portanto $\bar{\Sigma}_{k}=\bar{\Sigma}_{k_{0}}$, do qual segue imediatamente $P_{G}\left(\left.f_{k}\right|_{\Sigma_{k}}\right)=P_{G}\left(f_{k_{0}} \mid \bar{\Sigma}_{k_{0}}\right)$, além disso como $\bar{\Sigma}_{k}$ e $\bar{\Sigma}_{k_{0}}$ tem as mesmas componentes transitivas, então 
$N_{f_{k}}=N_{f_{k_{0}}}$, em consequência

$$
\sum_{J=1}^{N_{f_{k}}} \gamma_{J} \int g d \nu_{k, J}=\sum_{J=1}^{N_{f_{k_{0}}}} \gamma_{J} \int g d \nu_{k_{0}, J}
$$

ou seja para cada $k \geq k_{0}$,

$$
\lim _{t \rightarrow \infty} \int g d \mu_{t f_{k}}=\lim _{t \rightarrow \infty} \int g d \mu_{t f_{k_{0}}}
$$

Denotamos por

$$
\mu_{\infty}=\sum_{J=1}^{N_{f_{k_{0}}}} \gamma_{J} \nu_{k_{0}, J}
$$

Então para cada $k \geq k_{0}$ temos $\lim _{t \rightarrow \infty} \mu_{t f_{k}}=\mu_{\infty}$.

O lema anterior é basicamente uma versão mais forte do resultado obtido em [13] no sentido que temos convergência das sequências de estados de equilíbrio sobre cada subshift $\Sigma_{k}$ e além disso o limite coincide para cada $k \geq k_{0}$.

Para demonstrar o teorema B simplesmente é necessario fazer uma aproximação do limite em temperatura zero no subshift $\Sigma$ pelos limites em temperatura zero nos subshifts compactos $\Sigma_{k}$, o qual é uma consequência imediata do lema 17.

\section{Demonstração. (Teorema C)}

Seja $t>1$, by (5.1), o lema 14, Corolario 1 e o principio variacional (2.3) temos que existe o limite da sequência $\left(h\left(\mu_{t f_{k}}\right)\right)_{k \in \mathbb{N}}$ quando $k \rightarrow \infty$, mais ainda o limite é $h\left(\mu_{t f}\right)$. Além disso, dado que cada um dos $\Sigma_{k}$ 's com $k \in \mathbb{N}$ é um conjunto compacto, então cada uma das funções $t \mapsto h\left(\mu_{t f_{k}}\right)$ é decrescente, ou seja para cada $t_{1}>t_{0}>1$ e todo $k \in \mathbb{N}$ temos que $h\left(\mu_{t_{0} f_{k}}\right)>h\left(\mu_{t_{1} f_{k}}\right)$, portanto tomando o limite quando $k \rightarrow \infty$ segue que

$$
h\left(\mu_{t_{0} f}\right)=\lim _{k \rightarrow \infty} h\left(\mu_{t_{0} f_{k}}\right) \geq \lim _{k \rightarrow \infty} h\left(\mu_{t_{1} f_{k}}\right)=h\left(\mu_{t f}\right)
$$

em outras palavras a família $\left(h\left(\mu_{t f}\right)\right)_{t>1}$ é não crescente, particularmente para valores de $t$ suficientemente grandes a família é limitada.

Agora vamos mostrar que $\lim \sup _{t \rightarrow \infty} h\left(\mu_{t f}\right) \leq h\left(\mu_{\infty}\right)$ onde a medida de probabilidade $\mu_{\infty}$ é o limite na topologia fraca* de alguma subsequência convergente da família $\left(\mu_{t f}\right)_{t>1}$. De fato podemos observar que para cada sequência crescente $\left(t_{j}\right)_{j \in \mathbb{N}} \subset(1, \infty)$, e cada cilindro $[\omega] \in \alpha^{n_{0}}$, escolhendo 
$t^{\prime} \geq t_{j}$ segue que

$$
\inf _{t \geq t_{j}}\left\{-\mu_{t f}[\omega] \log \left(\mu_{t f}[\omega]\right)\right\} \leq-\mu_{t^{\prime} f}[\omega] \log \left(\mu_{t^{\prime} f}[\omega]\right)
$$

portanto para toda sequência crescente $\left(t_{j}\right)_{j \in \mathbb{N}} \subset(1, \infty)$ tal que existe o limite na topologia fraca* $\lim _{j \rightarrow \infty} \mu_{t_{j} f}=\mu_{\infty}$, somando sobre todos os cilindros $[\omega] \in \alpha^{n_{0}}$, temos que para cada $t^{\prime} \geq t_{j}$

$$
\sum_{[\omega] \in \alpha^{n_{0}}} \inf _{t \geq t_{j}}\left\{-\mu_{t f}[\omega] \log \left(\mu_{t f}[\omega]\right)\right\} \leq \sum_{[\omega] \in \alpha^{n_{0}}}-\mu_{t^{\prime} f}[\omega] \log \left(\mu_{t^{\prime} f}[\omega]\right)
$$

mais ainda como consequência imediata da ultima desigualdade temos que

$$
\sum_{[\omega] \in \alpha^{n_{0}}} \inf _{t \geq t_{j}}\left\{-\mu_{t f}[\omega] \log \left(\mu_{t f}[\omega]\right)\right\} \leq \inf _{t^{\prime} \geq t_{j}}\left\{\sum_{[\omega] \in \alpha^{n_{0}}}-\mu_{t^{\prime} f}[\omega] \log \left(\mu_{t^{\prime} f}[\omega]\right\}\right.
$$

Por outro lado se definimos o conjunto $X=\left\{[\omega]:[\omega] \in \alpha^{n_{0}}\right\}$ e a sequência de funções $\phi_{j}: X \rightarrow \mathbb{R}$ with $j \in \mathbb{N}$, onde $\phi_{j}([\omega])=\inf _{t \geq t_{j}}\left\{-\mu_{t f}[\omega] \log \left(\mu_{t f}[\omega]\right)\right\}$, denotando por $\delta$ à medida de contagem sobre o conjunto $X$, ou seja a medida satisfazendo $\int_{[\omega]} \phi_{j} d \delta=\phi_{j}([\omega])$ para todo $[\omega] \in \alpha^{n_{0}}$, então o pelo teorema de convergência monótona temos que $\lim _{j \rightarrow \infty} \int_{X} \phi_{j} d \delta=\int_{X} \lim _{j \rightarrow \infty} \phi_{j} d \delta$, ou em outras palavras

$$
\lim _{j \rightarrow \infty} \sum_{[\omega] \in \alpha^{n_{0}}} \inf _{t \geq t_{j}}\left\{-\mu_{t f}[\omega] \log \left(\mu_{t f}[\omega]\right)\right\}=\sum_{[\omega] \in \alpha^{n_{0}}} \lim _{j \rightarrow \infty} \inf _{t \geq t_{j}}\left\{-\mu_{t f}[\omega] \log \left(\mu_{t f}[\omega]\right)\right\}
$$

e dado que $\mu_{\infty}(\partial[\omega])=0$ para cada $[\omega] \in \alpha^{n_{0}}$ e temos existência do limite na topologia fraca* $\lim _{j \rightarrow \infty} \mu_{t_{j} f}=\mu_{\infty}$, então

$$
\lim _{j \rightarrow \infty} \inf _{t \geq t_{j}}\left\{-\mu_{t f}[\omega] \log \left(\mu_{t f}[\omega]\right)\right\}=\lim _{j \rightarrow \infty}-\mu_{t_{j} f}[\omega] \log \left(\mu_{t_{j} f}[\omega]\right)=-\mu_{\infty}[\omega] \log \left(\mu_{\infty}[\omega]\right)
$$

para todo $[\omega] \in \alpha^{n_{0}}$, em outras palavras a equação (5.12) é equivalente à equação

$$
\lim _{j \rightarrow \infty} \sum_{[\omega] \in \alpha^{n_{0}}} \inf _{t \geq t_{j}}\left\{-\mu_{t f}[\omega] \log \left(\mu_{t f}[\omega]\right)\right\}=\sum_{[\omega] \in \alpha^{n_{0}}}-\mu_{\infty}[\omega] \log \left(\mu_{\infty}[\omega]\right)
$$

Portanto tomando o limite quando $j \rightarrow \infty$ nos dois lados da equação (5.11) e usando (5.13) podemos concluir que

$$
\sum_{[\omega] \in \alpha^{n_{0}}}-\mu_{\infty}[\omega] \log \left(\mu_{\infty}[\omega]\right) \leq \liminf _{t \rightarrow \infty} \sum_{[\omega] \in \alpha^{n_{0}}}-\mu_{t f}[\omega] \log \left(\mu_{t f}[\omega]\right)
$$

ou em outras palavras $H\left(\mu_{\infty} \mid \alpha^{n_{0}}\right) \leq \liminf _{t \rightarrow \infty} H\left(\mu_{t f} \mid \alpha^{n_{0}}\right)$. Com uma demonstração similar tro- 
cando $\inf _{t \geq t_{j}}$ by $\sup _{t \geq t_{j}}$ e mudando as desigualdades acima, podemos mostrar facilmente que $\lim \sup _{t \rightarrow \infty} H\left(\mu_{t f} \mid \alpha^{n_{0}}\right) \leq H\left(\mu_{\infty} \mid \alpha^{n_{0}}\right)$, portanto podemos concluir que

$$
\lim _{t \rightarrow \infty} H\left(\mu_{t f} \mid \alpha^{n_{0}}\right)=H\left(\mu_{\infty} \mid \alpha^{n_{0}}\right) .
$$

Agora vamos mostrar que $\lim \sup _{t \rightarrow \infty} h\left(\mu_{t f}\right) \leq h\left(\mu_{\infty}\right)$. Suponhamos que não é verdade, ou seja $h\left(\mu_{\infty}\right)<\lim \sup _{t \rightarrow \infty} h\left(\mu_{t f}\right)$, então podemos escolher $\epsilon>0$ tal que $h\left(\mu_{\infty}\right) \leq \lim \sup _{t \rightarrow \infty} h\left(\mu_{t f}\right)-3 \epsilon$, mais ainda pela definição da entropia de Kolmogorov-Sinai para $n$ suficientemente grande $\frac{1}{n} H\left(\mu_{\infty} \mid\right.$ $\left.\alpha^{n}\right) \leq h\left(\mu_{\infty}\right)+\epsilon$, e pela equação (5.14) existe $n_{0} \geq n$ tal que $\lim _{t \rightarrow \infty} H\left(\mu_{t f} \mid \alpha^{n_{0}}\right)=H\left(\mu_{\infty} \mid \alpha^{n_{0}}\right)$. Finalmente pela definição de entropia de Kolmogorov-Sinai, podemos concluir que para cada $t>1$ é satisfeito $h\left(\mu_{t f}\right) \leq \frac{1}{n_{0}} H\left(\mu_{t f} \mid \alpha^{n_{0}}\right)$, portanto para $t$ suficientemente grande

$$
\begin{aligned}
h\left(\mu_{t f}\right) & \leq \frac{1}{n_{0}} H\left(\mu_{\infty} \mid \alpha^{n_{0}}\right)+\epsilon \\
& \leq h\left(\mu_{\infty}\right)+2 \epsilon \\
& \leq \limsup _{l \rightarrow \infty} h\left(\mu_{t f}\right)-\epsilon .
\end{aligned}
$$

Então tomando o limsup quando $t \rightarrow \infty$, obtemos uma contradição. Em conclusão

$$
\limsup _{t \rightarrow \infty} h\left(\mu_{t f}\right) \leq h\left(\mu_{\infty}\right)
$$

Por outro lado, para cada $k \geq k_{0}$ temos que $\mathcal{M}_{\max }\left(f_{k}\right)=\mathcal{M}_{\max }(f)$ e $\beta_{k}=\beta$, além disso pelo principio variacional (2.3) temos que

$$
P_{k}(t)-t \beta \geq \sup _{\nu \in \mathcal{M}_{\max }(f)} h(\nu) \geq h\left(\mu_{\infty}\right)
$$

onde $\mu_{\infty}$ é qualquer ponto de acumulação da família $\left(\mu_{t f}\right)_{t>1}$, portanto tomando o supremo sobre todos os valores de $k \geq k_{0}$, obtemos que

$$
\sup _{k \geq k_{0}}\left\{P_{k}(t)-t \beta\right\} \geq \sup _{\nu \in \mathcal{M}_{\max }(f)} h(\nu) \geq h\left(\mu_{\infty}\right) .
$$

Mais ainda, temos que $\sup _{k \geq k_{0}}\left\{P_{k}(t)-t \beta\right\}=\sup _{k \in \mathbb{N}}\left\{P_{k}(t)-t \beta\right\}=P(t)-t \beta$, portanto segue do principio variacional (2.3) e (5.16) que

$$
h\left(\mu_{t f}\right) \geq P(t)-t \beta \geq \sup _{\nu \in \mathcal{M}_{\max }(f)} h(\nu) \geq h\left(\mu_{\infty}\right)
$$


particularmente para todo $j \in \mathbb{N}$ temos que

$$
\inf _{t \geq t_{j}} h\left(\mu_{t_{j} f}\right) \geq \sup _{\nu \in \mathcal{M}_{\max }(f)} h(\nu) \geq h\left(\mu_{\infty}\right) .
$$

Então tomando o limite quando $j \rightarrow \infty$, obtemos que

$$
\liminf _{t \rightarrow \infty} h\left(\mu_{t f}\right)=\lim _{j \rightarrow \infty} \inf _{t \geq t_{j}} h\left(\mu_{t_{j} f}\right) \geq \sup _{\nu \in \mathcal{M}_{\max }(f)} h(\nu) \geq h\left(\mu_{\infty}\right) .
$$

Finalmente usando (5.15), podemos concluir que

$$
\liminf _{t \rightarrow \infty} h\left(\mu_{t f}\right) \geq \sup _{\nu \in \mathcal{M}_{\max }(f)} h(\nu) \geq h\left(\mu_{\infty}\right)=\limsup _{t \rightarrow \infty} h\left(\mu_{t f}\right),
$$

ou em outras palavras

$$
\lim _{t \rightarrow \infty} h\left(\mu_{t f}\right)=h\left(\mu_{\infty}\right) .
$$




\section{Capítulo 6}

\section{Conclusões}

No caso que $\Sigma$ é um subshift de Markov topologicamente transitivo e $f$ é um potencial somável, com pressão finita e $\bar{V}(f)<\infty$, podemos garantir a existência dos estados de equilíbrio $\mu_{t f}$ para cada $t>1$ e existência de um ponto de acumulação quando $t \rightarrow \infty$, além disso se o potencial $f$ é um potencial de Markov, ou seja $f(x)=f\left(x_{0}, x_{1}\right)$, também podemos garantir a existência do limite em temperatura zero.

A importância destes resultados é que fazemos uma generalização dos resultados que aparecem em [10] e [12] para um caso mais geral desde o ponto de vista dinâmico que é quando o sistema é topologicamente transitivo. Nós também obtemos uma generalização do resultado em [16] para o caso topologicamente transitivo.

Se o potencial não é Markov existem exemplos nos quais não existe o limite em temperatura zero, embora o subshift seja compacto, Chazottes e Hochman deram um exemplo de um potencial Lipschitz com dominio num subshift $\Sigma \subset\{0,1\}^{\mathbb{N}}$ para o qual não existe o limite em temperatura zero. Mais recentemente Coronel e Rivera-Letelier em [9] mostraram que se $\Sigma=\left(S^{1}\right)^{\mathbb{N}}$, dadas quaisquer duas medidas ergódicas com a mesma entropia é possível construir um potencial Lipschitz tal que a sequência de estados de equilíbrio acumula em medidas isomorfas às duas medidas.

Se o potencial não é somável, embora seja Markov não pode ser garantida a existência do limite em temperatura zero, um exemplo foi dado por Sarig em [21], nesse trabalho ele construí um potencial de Markov que não é somável para o qual não temos existência do limite em temperatura zero.

No caso que o $\Sigma$ é um subshift de Markov topologicamente transitivo com alfabeto enumerável demonstramos que existem pontos de acumulação da sequência $\left(\mu_{t f}\right)_{t>1}$ quando $t \rightarrow \infty$, além disso em [4] demonstraram que esses pontos de acumulação são de fato medidas maximizantes. Portanto 
uma questão em aberto que aparece como continuação natural deste trabalho é:

O principio dos grandes desvios é satisfeito para o caso de subshifts de Markov topologicamente transitivos com alfabeto enumerável? 


\section{Referências Bibliográficas}

[1] J. Aaronson. An introduction to infinite ergodic theory, volume 50 of Mathematical Surveys and Monographs. American Mathematical Society, Providence, RI, 1997. 7

[2] P. Billingsley. Convergence of probability measures. Wiley Series in Probability and Statistics: Probability and Statistics. John Wiley \& Sons, Inc., New York, second edition, 1999. A WileyInterscience Publication. 9

[3] R. Bissacot and R. Freire. On the existence of maximizing measures for irreducible countable Markov shifts: a dynamical proof. Ergodic Theory Dynam. Systems, 34(4):1103-1115, 2014. 5, 34,53

[4] R. Bissacot, J. K. Mengue, and E. Pérez. A large deviation principle for gibbs states on countable markov shifts at zero temperature. preprint, 2015. 59

[5] J. Brémont. Gibbs measures at temperature zero. Nonlinearity, 16(2):419-426, 2003. 3

[6] J. Buzzi and O. Sarig. Uniqueness of equilibrium measures for countable Markov shifts and multidimensional piecewise expanding maps. Ergodic Theory Dynam. Systems, 23(5):13831400, 2003. 5, 26

[7] J.-R. Chazottes, J.-M. Gambaudo, and E. Ugalde. Zero-temperature limit of one-dimensional Gibbs states via renormalization: the case of locally constant potentials. Ergodic Theory Dynam. Systems, 31(4):1109-1161, 2011. 3, 5, 37, 39

[8] J.-R. Chazottes and M. Hochman. On the zero-temperature limit of Gibbs states. Comm. Math. Phys., 297(1):265-281, 2010. 3, 54

[9] D. Coronel and J. Rivera-Letelier. Sensitive Dependence of Gibbs Measures at Low Temperatures. J. Stat. Phys., 160(6):1658-1683, 2015. 3, 59

[10] O. Jenkinson, R. D. Mauldin, and M. Urbański. Zero temperature limits of Gibbs-equilibrium states for countable alphabet subshifts of finite type. J. Stat. Phys., 119(3-4):765-776, 2005. $3,4,45,59$

[11] G. Keller. Equilibrium states in ergodic theory, volume 42 of London Mathematical Society Student Texts. Cambridge University Press, Cambridge, 1998. 37, 38

[12] T. Kempton. Zero temperature limits of Gibbs equilibrium states for countable Markov shifts. J. Stat. Phys., 143(4):795-806, 2011. 3, 4, 5, 37, 59

[13] R. Leplaideur. A dynamical proof for the convergence of Gibbs measures at temperature zero. Nonlinearity, 18(6):2847-2880, 2005. 3, 4, 5, 37, 39, 43, 44, 53, 54, 55

[14] R. D. Mauldin and M. Urbański. Gibbs states on the symbolic space over an infinite alphabet. Israel J. Math., 125:93-130, 2001. 3, 25 
[15] R. D. Mauldin and M. Urbański. Graph directed Markov systems, volume 148 of Cambridge Tracts in Mathematics. Cambridge University Press, Cambridge, 2003. Geometry and dynamics of limit sets. 7

[16] I. D. Morris. Entropy for zero-temperature limits of Gibbs-equilibrium states for countablealphabet subshifts of finite type. J. Stat. Phys., 126(2):315-324, 2007. 4, 5, 13, 43, 44, 59

[17] K. Oliveira and M. Viana. Fundamentos da Teoria Ergódica. 2016. 7, 9, 10, 12

[18] O. Sarig. Existence of Gibbs measures for countable Markov shifts. Proc. Amer. Math. Soc., 131(6):1751-1758 (electronic), 2003. 4

[19] O. Sarig. Lecture Notes on Thermodynamic Formalism for Topological Markov Shifts. Spring 2009. Penn State. 7, 14, 17, 19, 21, 31

[20] O. M. Sarig. Thermodynamic formalism for countable Markov shifts. Ergodic Theory Dynam. Systems, 19(6):1565-1593, 1999. 5, 14, 17, 18

[21] O. M. Sarig. On an example with a non-analytic topological pressure. C. R. Acad. Sci. Paris Sér. I Math., 330(4):311-315, 2000. 59

[22] O. M. Sarig. Phase transitions for countable Markov shifts. Comm. Math. Phys., 217(3):555577, 2001. 25 


\section{Índice Remissivo}

conjunto justo, 9

estado

de equilíbrio, 25

de Gibbs, 25

formula

Kâc, 18

Rokhlin, 18

medida

$R P F, 18$

conservativa, 8

ergódica, 9

invariante, 8

maximizante, 34

não-singular, 8

operador de Ruelle, 17

potencial

coercivo, 13

cohomologo, 13

Markov, 37

somável, 13

variação somável, 13

pressão de Gurevic, 14

teorema

decomposição ergódica, 11

ergódico de Birkhoff, 10

Livsic, 13

Prohorov, 9

recorrência de Poincaré, 9

unicidade dos estados de equilíbrio, 26

topologia fraca* ${ }^{*} 8$ 